\title{
Recent Advances in Electrochemical and Optical Sensors for Detecting Tryptophan and Melatonin
}

\author{
Zeeshan Ahmad Khan ${ }^{1-3}$ \\ Paul Jung-Soo Hong ${ }^{4, *}$ \\ Christina Hayoung Lee ${ }^{5, *}$ \\ Yonggeun Hong (D) ${ }^{1-3,6,7}$ \\ 'Department of Physical Therapy, College \\ of Healthcare Medical Science \& \\ Engineering, Inje University, Gimhae, \\ Gyeong-nam, 50834, Korea; ${ }^{2}$ Biohealth \\ Products Research Center (BPRC), Inje \\ University, Gimhae, Gyeong-nam, 50834, \\ Korea; ${ }^{3}$ Ubiquitous Healthcare \& Anti- \\ Aging Research Center (u-HARC), Inje \\ University, Gimhae, Gyeong-nam, 50834, \\ Korea; ${ }^{4}$ Department of Chemistry, \\ Newton South High School, Newton, \\ MA, 02459, USA; ${ }^{5}$ Department of \\ Biology, College of Arts and Sciences, \\ Vanderbilt University, Nashville, TN, \\ 37212, USA; ' Department of \\ Rehabilitation Science, Graduate School \\ of Inje University, Gimhae, Gyeong-nam, \\ 50834, Korea; ${ }^{7}$ Department of Medicine, \\ Division of Hematology/Oncology, \\ Harvard Medical School-Beth Israel \\ Deaconess Medical Center, Boston, MA, \\ 02215, USA
}

*These authors contributed equally to this work

Correspondence: Yonggeun Hong Department of Rehabilitation Science, Graduate School of Inje University, 197 Inje-ro, Gimhae, Gyeong-nam, 50834, Korea

Tel +82-55-320-368।

Fax +82-55-329-1678

Email yonghong@inje.ac.kr

\begin{abstract}
Tryptophan and melatonin are pleiotropic molecules, each capable of influencing several cellular, biochemical, and physiological responses. Therefore, sensitive detection of tryptophan and melatonin in pharmaceutical and human samples is crucial for human wellbeing. Mass spectrometry, high-performance liquid chromatography, and capillary electrophoresis are common methods for both tryptophan and melatonin analysis; however, these methods require copious amounts of time, money, and manpower. Novel electrochemical and optical detection tools have been subjects of intensive research due to their ability to offer a better signal-to-noise ratio, high specificity, ultra-sensitivity, and wide dynamic range. Recently, researchers have designed sensitive and selective electrochemical and optical platforms by using new surface modifications, microfabrication techniques, and the decoration of diverse nanomaterials with unique properties for the detection of tryptophan and melatonin. However, there is a scarcity of review articles addressing the recent developments in the electrochemical and optical detection of tryptophan and melatonin. Here, we provide a critical and objective review of high-sensitivity tryptophan and melatonin sensors that have been developed over the past six years (2015 onwards). We review the principles, performance, and limitations of these sensors. We also address critical aspects of sensitivity and selectivity, limit and range of detection, fabrication process and time, durability, and biocompatibility. Finally, we discuss challenges related to tryptophan and melatonin detection and present future outlooks.
\end{abstract}

Keywords: tryptophan, melatonin, electrochemical, sensors, voltammetry, optical

\section{Introduction}

Tryptophan and melatonin have been the subjects of scientific investigations for decades because of their pleiotropic activity, each capable of influencing several cellular, biochemical, and physiological responses. ${ }^{1,2}$ Tryptophan is considered an over-the-counter medication in numerous countries, crosses the blood-brain barrier efficiently, and acts as a nutritional supplement, sleep aid, appetite suppressant, and antidepressant. ${ }^{3}$ Tryptophan performs vital functions in the management of numerous medical conditions, like depression, obesity, cerebellar ataxia, persistent headaches, fibromyalgia, and insomnia. ${ }^{4-6}$ Owing to the diet dependency, biological impact, and therapeutic use, it is vital to accurately detect the level of tryptophan in food, pharmaceuticals, and human samples. Despite the essential nature, measurement of tryptophan has often been omitted in several studies, as the tryptophan analysis has generally been tedious. A key challenge of tryptophan detection is that the acidic condition used to hydrolyze protein for normal amino acid analysis causes substantial or even complete oxidative degradation of tryptophan. ${ }^{7}$ Alkaline hydrolysis is usually implemented to 
partially solve the problem of acid hydrolysis but it causes the formation of a high concentration of molecular oxygen that can interfere in the detection of tryptophan. Removing oxygen from the samples can be very time-consuming and, depending on the nature of the sample, very difficult. ${ }^{8}$

Tryptophan is an essential precursor of melatonin (5methoxy-N-acetyltryptamine) synthesis. The melatonin pathway is conserved and observed as tryptophan $\rightarrow 5$ hydroxytryptophan $\rightarrow$-hydroxytryptamine $\rightarrow \mathrm{N}$ -

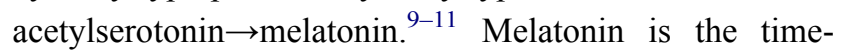
keeping molecule of vertebrates; it is synthesized in a circadian manner, enabling physiological, metabolic, and behavioral synchronization with the environmental lightdark cycle, light intensity, and temperature. ${ }^{12-14}$ As well as being a circadian regulator, melatonin is a potent antioxidant, immunoenhancer, and anti-inflammatory agent, osteoprotective, and is hence being considered as a therapeutic adjuvant for COVID-19 management and treatment. ${ }^{15-17}$ The daily levels of melatonin in human plasma range from a few pM during the day to $215-430 \mathrm{pM}$ at night. ${ }^{18,19}$ Determining melatonin levels has also become a means for diagnosing certain psychiatric disorders, and to recommend phase typing in patients suffering from sleep and mood disorders. ${ }^{20-22}$ Due to melatonin's low concentration in biological fluids, as well as the coexistence of numerous other compounds in the blood, the routine determination of melatonin has been an analytical challenge. ${ }^{23,24}$ Moreover, the large variations in melatonin levels through nature and products, as well as the varying complexities of the matrices in which melatonin is found, compel researchers to develop novel tools to extract melatonin before detection. Nonetheless, the selection of the extraction solvents is difficult due to the amphipathic and chemically reactive nature and of melatonin. ${ }^{23}$ Melatonin is also a commercial drug, which is available in the form of several types of pharmaceutical products such as modified-release tablets, prolonged-release tablets, regular tablets and capsules, and liquid medicine. The level of melatonin in pharmaceutical products ranges from 0.1 to $10 \mathrm{mg}$ per serving, ${ }^{25,26}$ and sensors offering micro and nanomolar sensitivity are used to measure melatonin in pharmaceutical products.

Over the last three decades, there has been a considerable increase in the need to measure tryptophan and melatonin levels in biological and pharmaceutical samples. Several novel and modified assays have been developed to investigate the physiological and psychological roles of tryptophan and melatonin, including high-performance liquid chromatography (HPLC), ${ }^{27-29}$ mass spectrometry, ${ }^{30,31}$ capillary electrophoresis, ${ }^{32,33}$ and gas chromatography. ${ }^{34,35}$ Although these methods have the benefits of sensitivity and accuracy, tryptophan and melatonin measurement tools are bulky, expensive, time-consuming, lacks portability, require laboratory maintenance, and skilled personnel. For reasons of cost and simplicity, radioimmunoassay (RIA) and enzyme-linked immunosorbent assay (ELISA) are most commonly used for melatonin detection. ${ }^{36}$ However, immunoassays are prone to show cross-reactivity with structurally similar compounds, which is a big challenge if the melatonin has not been extracted properly; full melatonin extraction is a complicated and time-consuming process. Electrochemical and optical detection of tryptophan and melatonin are inexpensive, offer sensitivity and portability, and are easy to operate, thereby, attracts researchers.

In recent years, there has been a steady rise in the development of numerous advanced functional nanomaterials-based sensors used in detecting tryptophan and melatonin. One such advanced functional material-developing tool is molecular imprinting technology (MIT) or molecular template technology, which is created for the purpose of synthesizing three-dimensional crosslinked polymers with specific molecular recognition ability. ${ }^{37}$ MIT-basedsensors offer the advantage of being relatively stable in storage, overcoming the key limitation of biological recognition elements such as enzymes or antibodies. ${ }^{38,39}$ However, the fabrication of MIT based-sensors is complex and needs expert handling. Moreover, MIT-sensors can recognize but cannot transmit signals out; therefore, they must be coupled with other methods such as an electrochemical or optical setup, which also have their own limitations. Another important advanced nanomaterialbased sensor is fluorescent "turn on-off" sensors. The fluorescent "turn on-off" showed promising capabilities for the detection of biomolecules due to their simplicity, fast response and high detection limits. Firstly, the redox potential of the analyte is exploited to change the oxidative state of functional groups on fluorophores, causing a switching "on" of the fluorescence of the dye. Subsequently, the "on" fluorophores were modified in a way where another redox agent binds to it, resulting in it switching "off". ${ }^{40}$ Although "turn on-off" sensors offer sensitivity and control, most of the conventional fluorophores with $\pi$-conjugation suffer from aggregationinduced quenching in biological samples resulting in a low fluorescence quantum yield, which limits their hightechnological application. ${ }^{41}$ 
In this review, we discuss the advantages and limitations of various electrochemical and optical tools for tryptophan and melatonin detection. The emphasis will be on human assays, although the application of assays for plants and pharmaceutical samples will be discussed where appropriate. The publications were collected from PubMed, Google Scholar, and Embase, and do not represent a systematic search. An overview of several emerging sensors (published in or after 2015) including electrochemical and optical sensors, and their related caveats was discussed (Tables 1 and 2). Finally, the challenges related to tryptophan and melatonin detection and their outlook in the future were presented.

\section{Electrochemical Detection of Tryptophan and Melatonin}

Electrochemical methods are promising as they are simple, easy to use, fast, require low-cost equipment, and offer comparatively good sensitivity and limit of detection (LOD). Moreover, electrochemical tools can be used to develop portable devices for in vivo and real-time sensing. Electrochemical detection consists of at least two electrodes (one working and one counter or auxiliary electrode) that are in contact with each other in two ways: an electrically conductive medium (electrolyte, ie liquid as an ion conductor) and an external electric circuit (electron conductor). The electrodes are made of special conducting material and have a catalytic effect so that in the presence of an analyte, certain chemical reactions can take place. ${ }^{42}$ Analytes are applied on the working electrode and the interaction of the analyte and electrode at a specific current or voltage causes an electrochemical reaction. This reaction is either an oxidation or a reduction reaction, depending on the type of analyte. Oxidation causes the flow of electrons from the working to the counter electrode through the external circuit. Reduction reaction transfer the flow of electrons from the counter to the working electrode. Either direction of the electron flow creates an electrical current proportional to the concentration of the analyte. Electrochemical detection can be performed by numerous electrodes and analytes in several ways depending on the different modes of applying electric potential, as well as current response models (Figure 1).

Voltammetry is an electroanalytical method in which current is measured as a function of applied potential to analyze chemicals and biomolecules qualitatively and quantitatively. Voltammetry reveals the reduction potential and electrochemical reactivity of an analyte, thereby facilitating analyte detection. Examples of common types of voltammetry include cyclic voltammetry (CV), linear sweep voltammetry (LSV), staircase voltammetry, square wave voltammetry (SWV), osteryoung square-wave stripping voltammetry (OSWSV), and differential pulse voltammetry (DPV). Voltammetry systems commonly comprise three electrodes: a reference electrode, a working electrode, and a counter electrode. In this system, an electrode may be modified with active materials or nanoparticles that can convert the ions of specific chemicals into measurable electrons at a particular voltage. The specific voltage is obtained by applying a potential to the electrode and sweeping the potential back and forth over a given voltage range for a defined number of cycles. The peak potential, at which the peak current is obtained, enables the user to analyze the electrochemical reversibility of the reaction at the electrode surface by varying the scan rates. ${ }^{43,44}$ In the case of $\mathrm{CV}$, the peak potentials vary linearly with time. On the other hand, the peak potentials of SWV and DPV represent constant increase in either square oscillations or rectangular pulses, respectively. ${ }^{45}$ Additionally, the three-electrode system may also be applied to characterize the redox properties, stability, and effective surface area of the modified electrode ${ }^{43,44}$ To make a comprehensive and objective evaluation of the analyte, it is necessary to select the most suitable electrochemical detection method, according to the features of the analyte including charge, electrocatalytic potential, redox potential, matrix, and size. For instance, through contrastive experiments, Gholivand et a ${ }^{46}$ compared the effects of $\mathrm{CV}$, LSV, and DPV in detecting bovine serum albumin (BSA). In the process, they have discovered that the DPV is the most suitable method for BSA detection, and applied the experimental results to clinical treatment. It is important to note that selection of the voltammetry technique is also dependent on the redox properties, shape, and size of the electrode too. Therefore, for the same analyte, a different voltammetry tool might be suitable depending on the type of electrode used. Also, the voltammetric tool can be selected depending upon the rate of reaction between electrode and analyte. For example, DPV is usually applied in irreversible systems and in systems that present slow-reaction kinetics. On the other hand, SWV is usually applied in reversible systems and rapid reaction kinetics systems.

\section{CV-Based Detection of Tryptophan}

$\mathrm{CV}$ is a versatile technique with applications for both direct and indirect analysis in many research areas involving electron transfer processes. ${ }^{47}$ Recently, Zhang et al ${ }^{48}$ developed 
Table I Recently Developed (After 2015) Electrochemical and Optical Sensors for Tryptophan

\begin{tabular}{|c|c|c|c|c|c|}
\hline $\begin{array}{l}\text { Sensing } \\
\text { Modality }\end{array}$ & Electrodes/Remarks & $\begin{array}{l}\text { Standard } \\
\text { Range }\end{array}$ & LOD & $\begin{array}{l}\text { Sample/Matrix; Analyte (s) (in } \\
\text { Addition to Tryptophan) }\end{array}$ & References \\
\hline $\mathrm{CV}$ & Carbon/nafion/GCE & $\begin{array}{l}0.5-70.0 \\
\mu \mathrm{M}\end{array}$ & $\begin{array}{l}35.0 \\
\mathrm{nM}\end{array}$ & $\begin{array}{l}\text { PST solution and tryptophan } \\
\text { spiked in mixture of amino acids }\end{array}$ & [48] \\
\hline \multirow[t]{5}{*}{ DPV } & $\begin{array}{l}\text { Screen-printed electrodes modified with carbon } \\
\text { nanotubes }\end{array}$ & $\begin{array}{l}0.005-3 \\
\mathrm{mM}\end{array}$ & $\begin{array}{l}4.9 \\
\mathrm{nM}\end{array}$ & PST solution & [58] \\
\hline & $\begin{array}{l}\text { Carbon paste electrode modified with } \mathrm{SnO}_{2}- \\
\mathrm{Co}_{3} \mathrm{O}_{4} @ \mathrm{rGO} \text { nanocomposite }\end{array}$ & $\begin{array}{l}0.02-6.00 \\
\mu M\end{array}$ & $\begin{array}{l}3.2 \\
\mathrm{nM}\end{array}$ & $\begin{array}{l}\text { PST and tryptophan spiked human } \\
\text { serum }\end{array}$ & [62] \\
\hline & Palladium-copper-copper oxide & $\begin{array}{l}0.02-25 \\
\mathrm{nM}\end{array}$ & $1.9 \mathrm{nM}$ & $\begin{array}{l}\text { PST and tryptophan spiked human } \\
\text { serum }\end{array}$ & [7I] \\
\hline & Tin oxide nanoparticles & $\mathrm{I}-100 \mu \mathrm{M}$ & $\begin{array}{l}0.04 \\
\mu \mathrm{M}\end{array}$ & $\begin{array}{l}\text { PST and tryptophan spiked human } \\
\text { serum }\end{array}$ & [72] \\
\hline & $\begin{array}{l}\text { Reduced graphene oxide-carboxymethyl cellulose- } \\
\text { polyaniline }\end{array}$ & $0.01-5 \mathrm{mM}$ & $\begin{array}{l}0.07 \\
\mu \mathrm{M}\end{array}$ & L- and D-tryptophan solution & [78] \\
\hline \multirow[t]{4}{*}{ sWV } & $\begin{array}{l}\text { 2-chlorobenzoylferrocene/ Ag- } \mathrm{ZnO} \text { nanoplates- } \\
\mathrm{CPE}\end{array}$ & $\begin{array}{l}0.05-20.0 \\
\mu M\end{array}$ & $\begin{array}{l}0.02 \\
\mu M\end{array}$ & PST solution & [103] \\
\hline & $\mathrm{Cu}_{2} \mathrm{O}-\mathrm{rGO} / \mathrm{GCE}$ & $\begin{array}{l}0.02-20 \\
\mu \mathrm{M}\end{array}$ & $\begin{array}{l}0.01 \\
\mu M\end{array}$ & $\begin{array}{l}\text { PST and tryptophan spiked human } \\
\text { serum }\end{array}$ & {$[111]$} \\
\hline & $\begin{array}{l}\mathrm{CuO} \text { nanoflakes anchored with polythiophene- } \\
\mathrm{GCE}\end{array}$ & $\begin{array}{l}0.1 \mu \mathrm{M}-\mathrm{I} \\
\mathrm{mM}\end{array}$ & $15 \mathrm{nM}$ & $\begin{array}{l}\text { PST and tryptophan spiked human } \\
\text { serum }\end{array}$ & [118] \\
\hline & $\begin{array}{l}\text { Nickel oxide and Copper oxide and citrate } \\
\text { modified GR on GCE }\end{array}$ & $0.3-40 \mu \mathrm{M}$ & $\begin{array}{l}0.1 \\
\mu M\end{array}$ & $\begin{array}{l}\text { PST and tryptophan spiked human } \\
\text { serum; dopamine, and } \\
\text { acetaminophen }\end{array}$ & [109] \\
\hline \multirow[t]{2}{*}{ Amperometry } & Dandelion pappus and drop-casted on GCE & $\begin{array}{l}\mathrm{I} \mu \mathrm{M}-10 \\
\mathrm{mM}\end{array}$ & $0.5 \mu \mathrm{M}$ & $\begin{array}{l}\text { PST, tryptophan spiked amino acid } \\
\text { solution and fetal calf serum }\end{array}$ & [133] \\
\hline & $\begin{array}{l}\text { Flow-injection; Silver nanoparticle on Screen- } \\
\text { printed electrodes }\end{array}$ & $\begin{array}{l}0.5 \mu \mathrm{M}-5 \\
\mathrm{mM}\end{array}$ & N.A. & PST solution & [138] \\
\hline Stochastic & $\begin{array}{l}\text { Nitrogen-doped graphene, } 2.2 \text {-diphenyl-I- } \\
\text { picrylhydrazyl, exfoliated graphene, protoporphyrin } \\
\text { IX and nGR }\end{array}$ & $\begin{array}{l}\mathrm{I} f \mathrm{M}-0.10 \\
\mathrm{mM}\end{array}$ & $I \mathrm{fM}$ & $\begin{array}{l}\text { PST solution and tryptophan in } \\
\text { whole blood }\end{array}$ & [148] \\
\hline \multirow[t]{6}{*}{ Optical } & Indole-hydroxylamine chemistry & $\begin{array}{l}100-600 \\
\mu \mathrm{M}\end{array}$ & $\begin{array}{l}100 \\
\mu M\end{array}$ & $\begin{array}{l}\text { PST, tryptophan with tyrosine and } \\
\text { phenylalanine }\end{array}$ & {$[151]$} \\
\hline & Metal-organic framework-ZJU-108 & N.A. & $\begin{array}{l}42.9 \\
\mathrm{nM}\end{array}$ & $\begin{array}{l}\text { PST and tryptophan spiked human } \\
\text { serum }\end{array}$ & {$[152]$} \\
\hline & Diazotization of naphthalene ethylenediamine & $\begin{array}{l}50 \mathrm{nM}-\mathrm{I} \\
\mathrm{mM}\end{array}$ & $20 \mathrm{nM}$ & $\begin{array}{l}\text { PST and tryptophan spiked human } \\
\text { serum }\end{array}$ & {$[154]$} \\
\hline & Phenylalanine derivative fluorescence sensor & $0-200 \mathrm{mM}$ & N.A. & PST solution; phenylalaninol & {$[157]$} \\
\hline & Chiral carbazole sensor & N.A. & $\begin{array}{l}0.31 \\
\mu M\end{array}$ & PST solution; histidine & [158] \\
\hline & Chitosan-capped silver nanoparticles & $13-460 \mu \mathrm{M}$ & $\begin{array}{l}2.1 \\
\mu M\end{array}$ & $\begin{array}{l}\text { PST and tryptophan spiked human } \\
\text { serum }\end{array}$ & [159] \\
\hline
\end{tabular}

Notes: This table includes the mode of sensing, features of assay including electrodes, standard range, the limit of detection (LOD), and sample/matrix, analyte(s), and references. Abbreviations: CV, cyclic voltammetry; DPV, differential pulse voltammetry; CPE, carbon paste electrode; GCE, glassy carbon electrode; PST, pharmaceutical samples of tryptophan; SWV, square wave voltammetry. This is not an exhaustive list. 


\begin{tabular}{|c|c|c|c|c|c|c|c|c|c|c|c|c|c|c|c|c|}
\hline 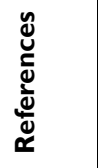 & 垔 & $\sqrt[\widetilde{n}]{6}$ & 물 & $\overline{\bar{\infty}}$ & $\underset{\widetilde{S}}{\overparen{S}}$ & 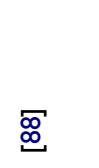 & F & $\bar{\Xi}$ & $\underset{\widetilde{D}}{\widetilde{\infty}}$ & 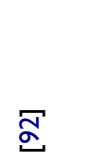 & $\underset{\infty}{\Phi}$ & $\frac{\sigma}{\Xi}$ & হ̄ं & 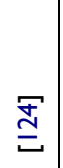 & 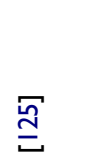 & $\stackrel{\text { ò }}{\underset{U}{U}}$ \\
\hline 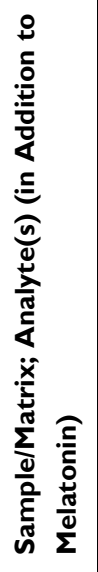 & 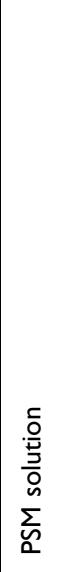 & 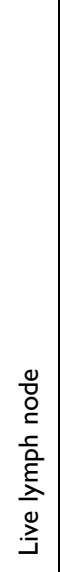 & 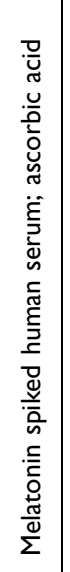 & 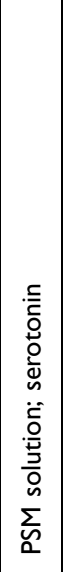 & 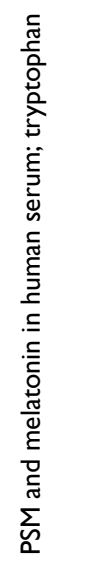 & 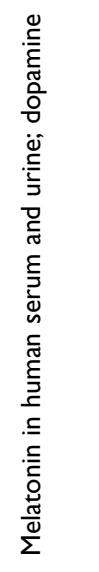 & 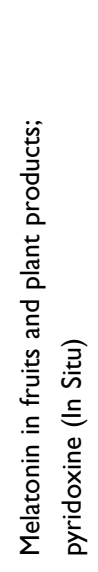 & 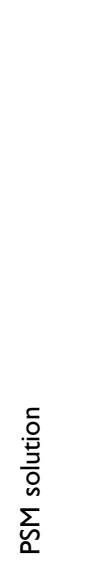 & 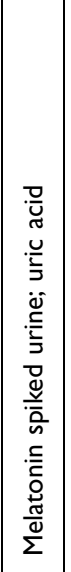 & 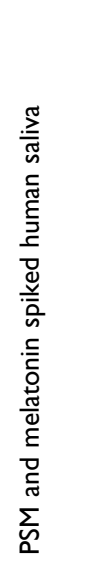 & 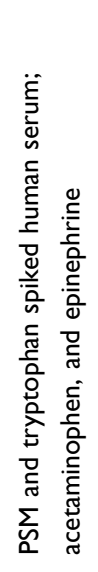 & 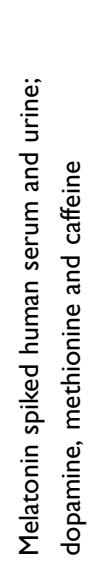 & 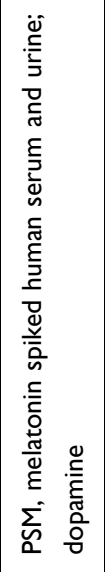 & 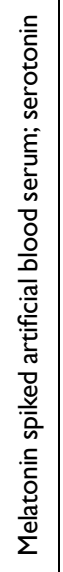 & 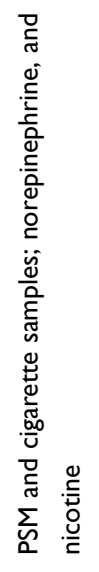 & 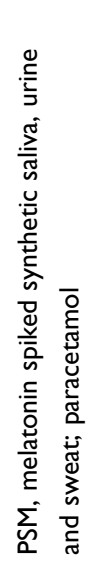 \\
\hline 。ิ & $\begin{array}{c}\sum_{c} \\
\stackrel{0}{\infty} \\
\infty\end{array}$ & 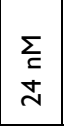 & $\begin{array}{l}\Sigma \\
\Sigma \\
\varnothing \\
\wp\end{array}$ & $\begin{array}{l}\Sigma \\
\Xi \\
\Xi \\
\end{array}$ & $\frac{\sum_{c}}{\dot{\sigma}}$ & $\frac{\sum}{\Sigma}$ & $\begin{array}{l}\sum_{c} \\
\underline{0}\end{array}$ & $\begin{array}{l}\sum_{c} \\
\hat{i}\end{array}$ & $\begin{array}{c}\sum_{c} \\
\underline{n} \\
\underline{\underline{n}} \\
\end{array}$ & $\begin{array}{l}\sum_{c} \\
\Lambda \\
\end{array}$ & $\sum_{\Xi}$ & $\begin{array}{l}\sum \\
\text { 三 } \\
\text { 员 } \\
\text { 人 }\end{array}$ & $\begin{array}{l}\sum_{c} \\
0 \\
\phi \\
\infty\end{array}$ & $\begin{array}{l}\sum_{\Xi} \\
\sigma \\
\underline{-}\end{array}$ & 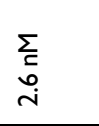 & 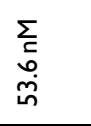 \\
\hline 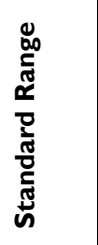 & $\begin{array}{l}\sum_{I} \\
8 \\
0 \\
I \\
I\end{array}$ & $\left|\begin{array}{l}\sum \\
\Xi \\
0 \\
1 \\
\sum_{c} \\
0 \\
i n\end{array}\right|$ & $\left|\begin{array}{c}\Sigma \\
\xi \\
0 \\
i \\
i \\
\Sigma \\
\Sigma \\
\tilde{I} \\
\tilde{O} \\
0\end{array}\right|$ & $\left|\begin{array}{c}\Sigma \\
\varepsilon \\
m \\
1 \\
\stackrel{0}{0} \\
0 \\
0\end{array}\right|$ & $\begin{array}{l}\sum_{\Xi} \\
8 \\
0 \\
0 \\
\grave{1} \\
0 \\
0\end{array}$ & $\begin{array}{l}\sum_{3} \\
\delta \\
0 \\
0 \\
\vdots \\
0 \\
0\end{array}$ & 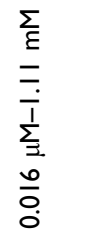 & $\begin{array}{l}\sum_{\Xi} \\
8 \\
\varnothing \\
\infty \\
0 \\
0\end{array}$ & $\left|\begin{array}{l}\Sigma \\
\vdots \\
0 \\
0 \\
0 \\
\tilde{m} \\
\tilde{0} \\
0 \\
0\end{array}\right|$ & 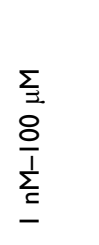 & 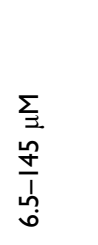 & 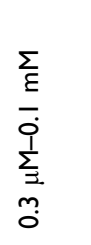 & $\begin{array}{l}\Sigma \\
\Sigma \\
0 \\
o \\
0 \\
\Lambda \\
\Lambda \\
\delta \\
0 \\
0\end{array}$ & 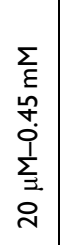 & $\begin{array}{l}\frac{\sum}{\varepsilon} \\
\frac{1}{1} \\
\sum_{\Xi} \\
0 \\
0 \\
0\end{array}$ & 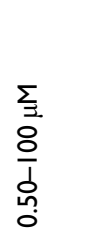 \\
\hline 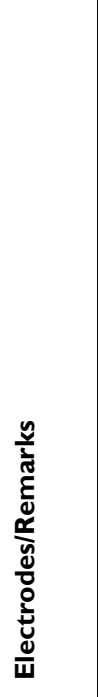 & 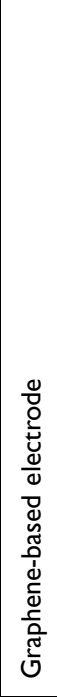 & 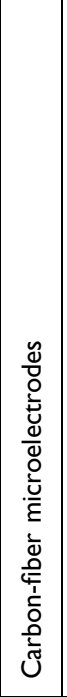 & 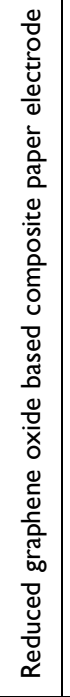 & 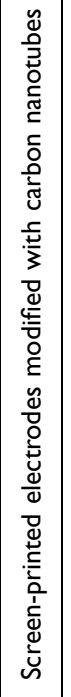 & 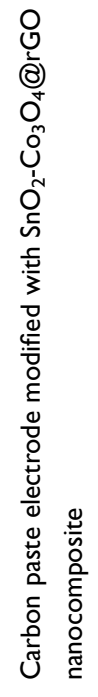 & 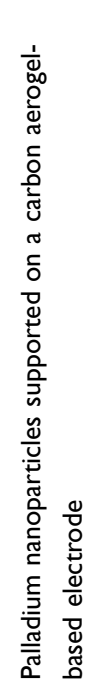 & 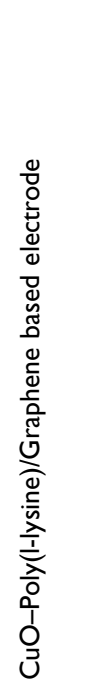 & 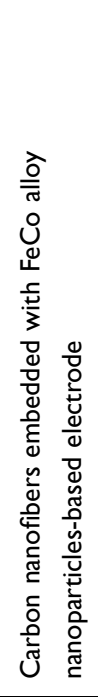 & 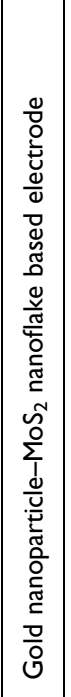 & 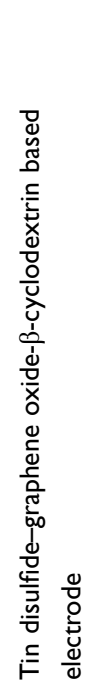 & 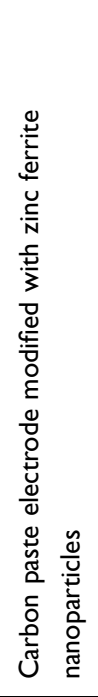 & 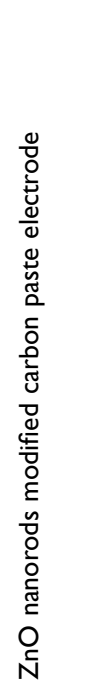 & 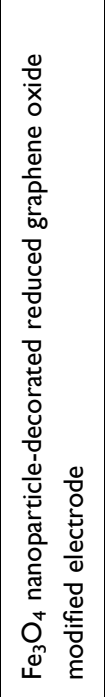 & 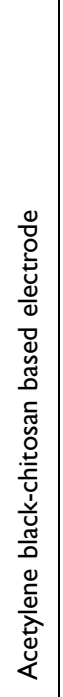 & 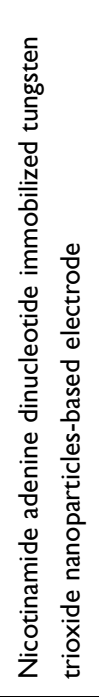 & 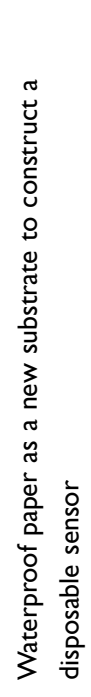 \\
\hline 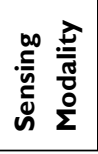 & U & & & a & & & & & & & & 交 & & & & \\
\hline
\end{tabular}




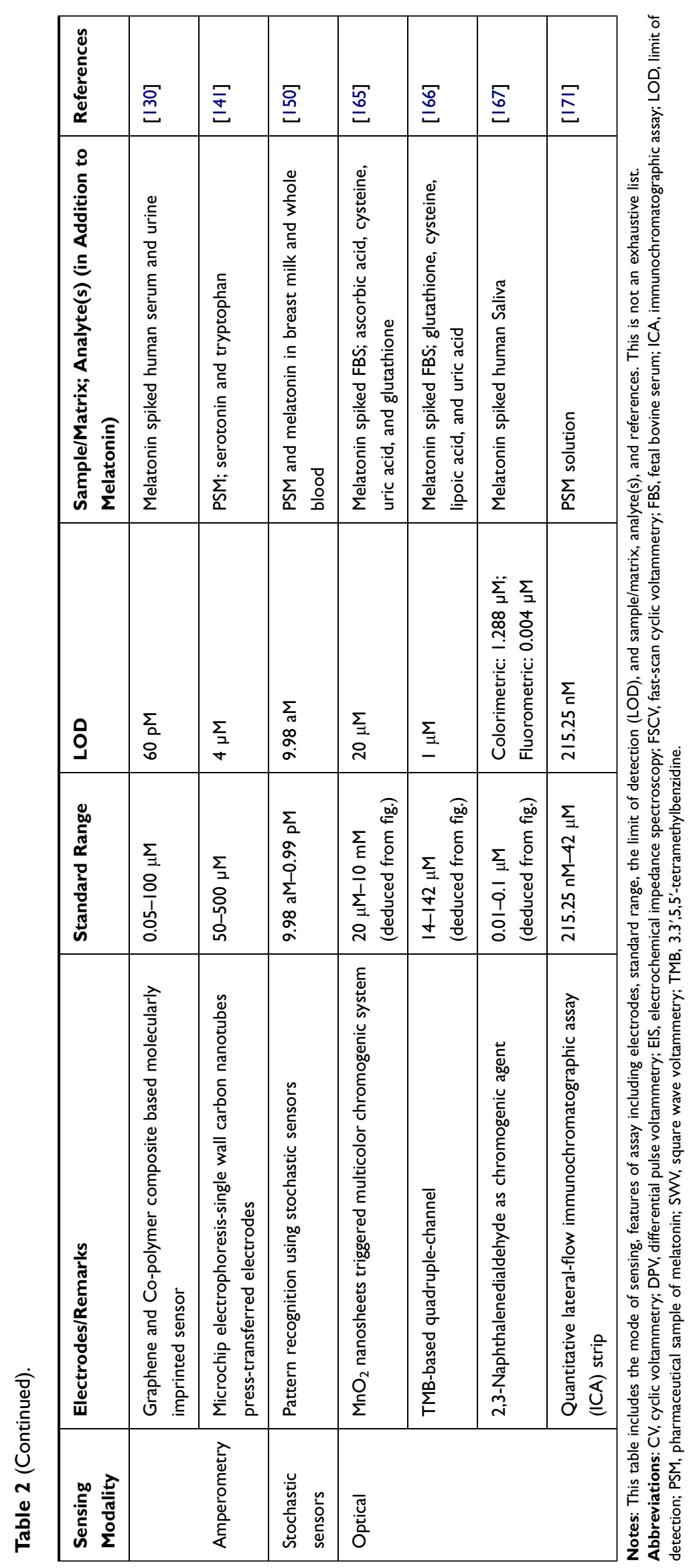




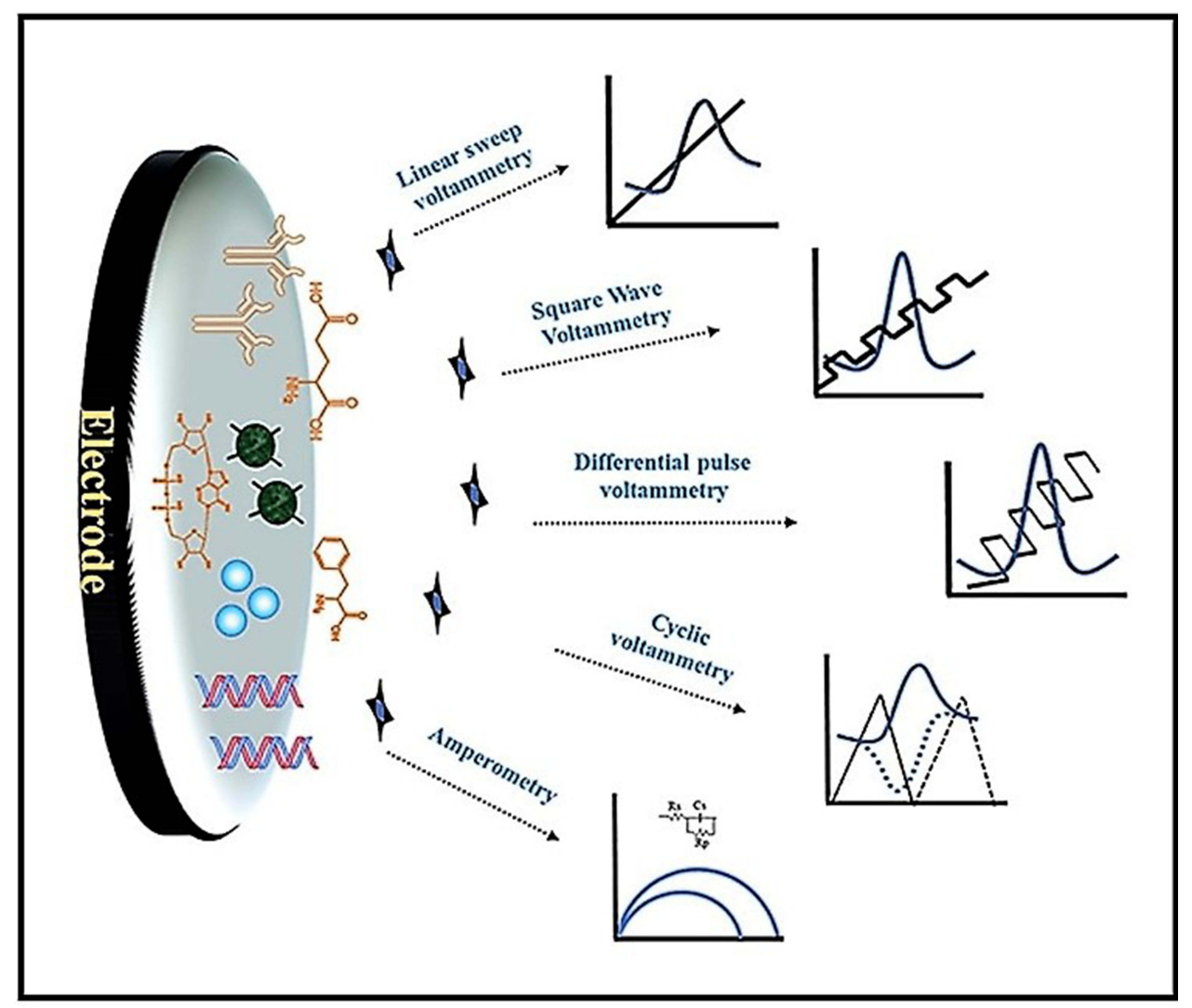

Figure I Electrochemical technique for detection of tryptophan and melatonin. Schematic representation of an electrochemical sensor, and the transduction of these interactions into measurable signals. The star $(\phi)$ represents the analyte (melatonin/tryptophan). The black line ( (

an electrochemical sensor containing nitrogen-doped ordered mesoporous carbon/Nafion/glassy carbon electrode (GCE) for the highly sensitive and selective detection of tryptophan. Cyclic voltammogram exhibited a linear increase in the oxidation current with tryptophan concentration in the range of $0.5-70.0 \mu \mathrm{M}$, with a LOD of $35.0 \mathrm{nM}$. In order to check the selectivity, tryptophan was measured in a mixture of 18 amino acid, which showed excellent recoveries in the range of $99.30-103.60 \%$. Although the sensor was highly sensitive and selective for tryptophan, fabrication was extremely time-consuming and can take up to 6 days. Furthermore, this paper lacked physical, chemical, or microstructure characterization of the modified materials, thereby, making it difficult to understand the reason behind the high sensitivity and selectivity.

\section{CV-Based Detection of Melatonin}

Cyclic voltammetry measurements can also be performed using a graphene-coated screen-printed carbon electrode
(G-SPE) to assess melatonin levels in pharmaceutical products. ${ }^{49}$ Graphene coating provided a large surface area (2630 $\mathrm{m}^{2} / \mathrm{g}$ for a single layer, theoretically), exceptional thermal conductivity $(\mathrm{k}=5000 \mathrm{~W} / \mathrm{mK})$, and electrical conductivity $(\mathrm{r}=64 \mathrm{mS} / \mathrm{cm}) ;{ }^{50,51}$ this led to a high number of available electroactive sites, thus enhancing the sensitivity of the carbon electrode. For this graphene-based electrochemical sensor, the linear detection range was $1-300 \mu \mathrm{M}$ and the LOD was $870 \mathrm{nM}$. The main limitation of this study was that it did not address potential interference from indoles that are structurally and chemically similar to melatonin, such as tryptophan and serotonin. Hence, G-SPE cannot be recommended for the evaluation of melatonin in complex biological samples that usually contain higher levels of tryptophan and serotonin than melatonin.

The in vivo detection of melatonin is still a significant challenge. To address this, Hensley et $\mathrm{a}^{52}$ developed an electrochemical technique for melatonin detection in live lymph nodes; they achieved subsecond temporal resolution by using 
fast-scan cyclic voltammetry (FSCV) with carbon-fiber microelectrodes (CFMEs). Initially, the oxidation peaks detected for melatonin were at 1.0, 1.1, and $0.6 \mathrm{~V}$. However, a tertiary peak due to electrode fouling was also observed. Bio-fouling is a major challenge for assays using electrochemical sensors, especially for in vivo assays. This is because the oxidation products of biological analytes can electropolymerize in solution and absorb onto the electrode surface, leading to the detection of [wrong] peaks during electrochemical evaluations. Live tissues can further aggravate this issue by physically attaching to the electrode. The biofouling interference issue was overcome by developing a customized waveform. The optimized waveform for melatonin consisted of scanning from 0.2 to $1.3 \mathrm{~V}$ and back at $600 \mathrm{~V} / \mathrm{s}$; this reduced the signal produced by the electrode fouling while maintaining the sensitivity level. The LOD obtained with this method using CFMEs and FSCV was $24 \pm 10 \mathrm{nM}$ for melatonin. Moreover, the CFME-FSCV method could successfully detect melatonin in the presence of biological interferences and could co-detect melatonin alongside its biosynthetic precursor, serotonin. This method was then validated by successfully measuring melatonin levels in intact lymph node tissue, making this the first report of FSCV usage for analyzing this tissue type. Despite this accomplishment, the durability of CFMEs constitutes an area of concern. Due to the fragile nature of CFMEs, these microelectrodes are prone to damage during in vivo analyses. Therefore, instead of using microelectrodes made of carbon fiber, applying a carbon coating to metal electrodes could yield the desired durability while keeping the biocompatible and electrochemical properties of carbon. Additionally, arrays of CFMEs/carbon-coated metal microelectrodes could be developed for the simultaneous measurement of multiple analytes, such as melatonin biosynthetic precursors (eg, tryptophan, serotonin, and $\mathrm{N}$-acetylserotonin); this may provide useful insight into the regulation of melatonin synthesis in vivo.

The electrochemical detection of melatonin is mostly performed using solid, rigid electrodes, such as carbon paste electrodes (CPEs), boron-doped diamond electrodes, and GCEs. ${ }^{53-55}$ Very recently, a flexible graphene-based paper composed of boron oxide $\left(\mathrm{B}_{2} \mathrm{O}_{3}\right)$ and graphene was developed using vacuum filtration, then used to detect melatonin and ascorbic acid (AA) simultaneously in spiked serum samples. ${ }^{56}$ To generate the paper, $\mathrm{B}_{2} \mathrm{O}_{3}$ nanostructures were manufactured using a hydrothermal method, then a boron-graphene oxide (B-GO) composite was synthesized by mixing $\mathrm{B}_{2} \mathrm{O}_{3}$ in a $\mathrm{GO}$ dispersion. $\mathrm{B}_{2} \mathrm{O}_{3}$-reduced graphene oxide (B-rGO) paper was procured via vacuum filtration of the $\mathrm{B}-\mathrm{GO}$ dispersion, then chemical reduction of the filtrate with 57\% (w/w) hydroiodic acid (HI) (Figure 2). Although

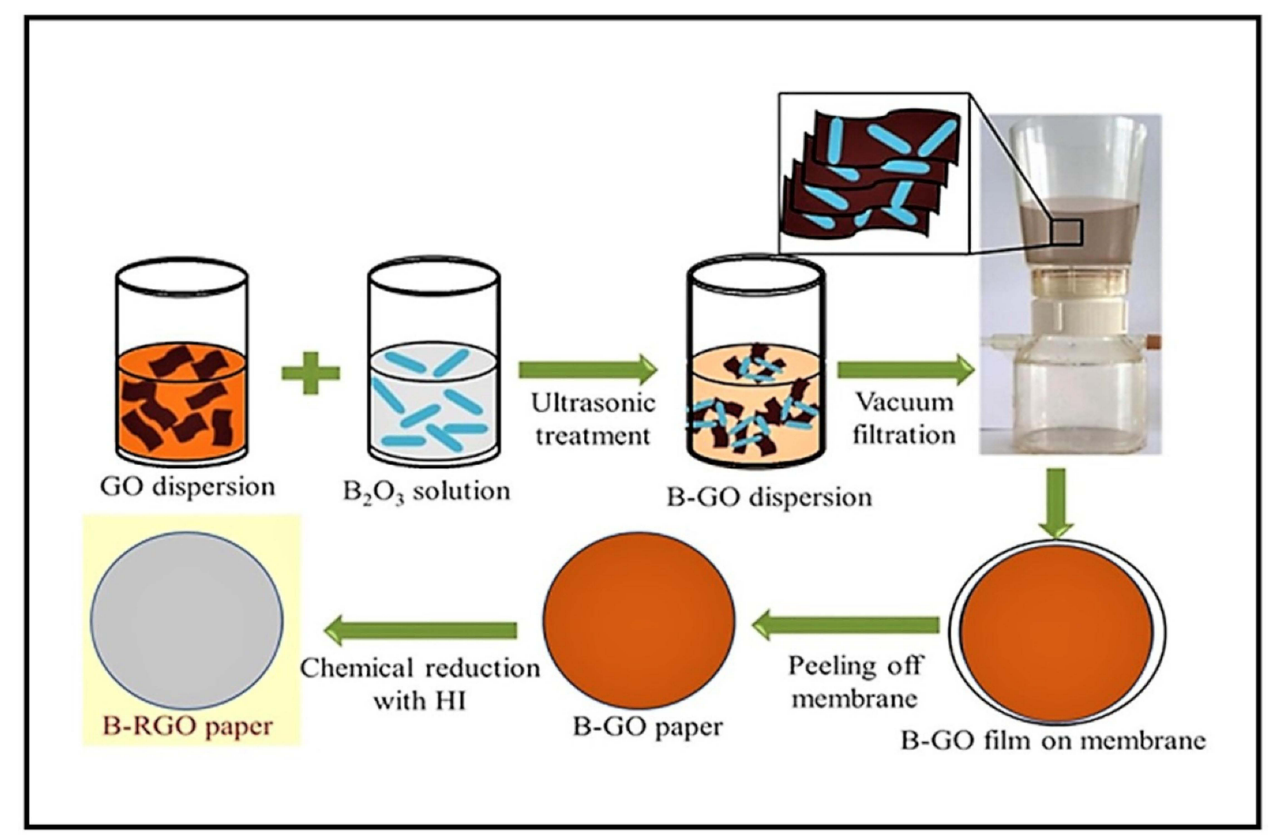

Figure 2 Schematic representation of boron oxide-reduced graphene oxide (B-rGO) composite paper fabrication.

Notes: Graphene oxide (GO) was dispersed and mixed with boron oxide $\left(\mathrm{B}_{2} \mathrm{O}_{3}\right)$ solution then sonicated. The $\mathrm{B}_{2} \mathrm{O}_{3}$ - $\mathrm{GO}$ composite $(\mathrm{B}-\mathrm{GO})$ was then vacuum filtrated then dried to form $\mathrm{B}-\mathrm{GO}$ paper. The $\mathrm{B}-\mathrm{GO}$ paper was next immersed in hydrochloric acid $(\mathrm{HI})$ solution, then washed and air-dried to generate the $\mathrm{B}_{2} \mathrm{O}_{3}-\mathrm{reduced} \mathrm{GO}(\mathrm{B}-\mathrm{rGO})$ composite paper. Adapted from Diamond and Related Materials, Vol 105, Issue 2020, Topçu E, Dağcı Kıranșan K, Electrochemical simultaneous sensing of melatonin and ascorbic acid at a novel flexible B-RGO composite paper electrode, Page No. 1078II, Copyright (2020), with permission from Elsevier. ${ }^{56}$ 
the B-rGO paper electrode was flexible, durable, and could be molded into the desired shape, the laborious production of B-rGO composite paper, as well as the use of toxic, strongly corrosive, and environmentally unfriendly $\mathrm{HI}$, presented major limitations to this work. Moreover, the LOD of this paper-based melatonin sensor was $700 \mathrm{nM}$, which limits its applicability.

\section{DPV-Based Simultaneous Detection of Tryptophan and Melatonin}

The primary advantage of the DPV is its enhanced capability to distinguish the faradaic (charging/capacitance) current, thereby minimizes background charging currents. As a result, sensors employing DPV are more sensitive to charging currents (electron transfer to and from an electrode) as compared to conventional sampled DC voltammetry. DPV generates small peaks of faradaic currents superimposed upon a staircase waveform, as opposed to sigmoidal waveforms; this results in improved resolution, low background, and better analyte quantitation in multiple analyte systems. ${ }^{57}$ Therefore, DPV is a promising tool for both individual and simultaneous molecule detection.

In 2015, a DPV-based sensor was developed for the simultaneous detection of melatonin and tryptophan by Tadayon et al. ${ }^{58}$ To simultaneously detect multiple analytes, each analyte in the suspension must be oxidized at a specific oxidation potential. Therefore, graphene was utilized as a base material due to its high electrical conductivity and surface area. In order to further improve the electrocatalytic activity for the simultaneous detection in the aforementioned studies, the surfaces of the electrodes were modified with a novel composite of graphene and copper. They constructed CPEs decorated with nitrogendoped graphene nanosheets/copper cobaltite $\left(\mathrm{CuCo}_{2} \mathrm{O}_{4}\right)$ nanoparticles, with which they achieved a LOD of $4.9 \mathrm{nM}$ for melatonin and $4.1 \mathrm{nM}$ for tryptophan. The sensitivity of the $\mathrm{CuCo}_{2} \mathrm{O}_{4}$ sensor for tryptophan was good enough to be used for the biological samples. However, to perform the simultaneous detection of melatonin and its biosynthetic precursors in biological samples, the sensitivity for melatonin detection should be enhanced to be applied to biological samples. Moreover, graphene toxicity is a major concern for human and environmental health. ${ }^{59-61}$

A more sensitive tool was later developed by Zeinali et $\mathrm{al}^{62}$ who employed a novel DPV-based electrochemical sensor to co-detect tryptophan and melatonin in interfering environments, such as urine and human serum. Their sensor was an ionic liquid (IL) CPE, whose surface was modified with $\mathrm{rGO}$ and tin oxide-cobalt oxide $\left(\mathrm{SnO}_{2^{-}}\right.$ $\left.\mathrm{Co}_{3} \mathrm{O}_{4}\right) \quad$ nanoparticles $\left(\mathrm{SnO}_{2}-\mathrm{Co}_{3} \mathrm{O}_{4} @ \mathrm{rGO} / \mathrm{IL} / \mathrm{CPE}\right)$ (Figure 3). ${ }^{62}$ Using the novel $\mathrm{SnO}_{2}-\mathrm{Co}_{3} \mathrm{O}_{4} @ \mathrm{rGO} / \mathrm{IL} / \mathrm{CPE}$ sensors, Zeinali et $\mathrm{al}^{62}$ demonstrated that during DPV oxidation of the analytes, the melatonin underwent a two-electron/one-proton reaction, while tryptophan underwent a two-electron/two-proton reaction; this enabled the selective sensing of the two biomolecules (Figure $3 \mathrm{C}-\mathrm{E}$ ). The large electroactive surface area and good electrical conductivity of $\mathrm{SnO}_{2}-\mathrm{Co}_{3} \mathrm{O}_{4} @ \mathrm{rGO} / \mathrm{IL} / \mathrm{CPEs}$ generated strong electrochemical responses during both melatonin measurements and melatonin/tryptophan co-

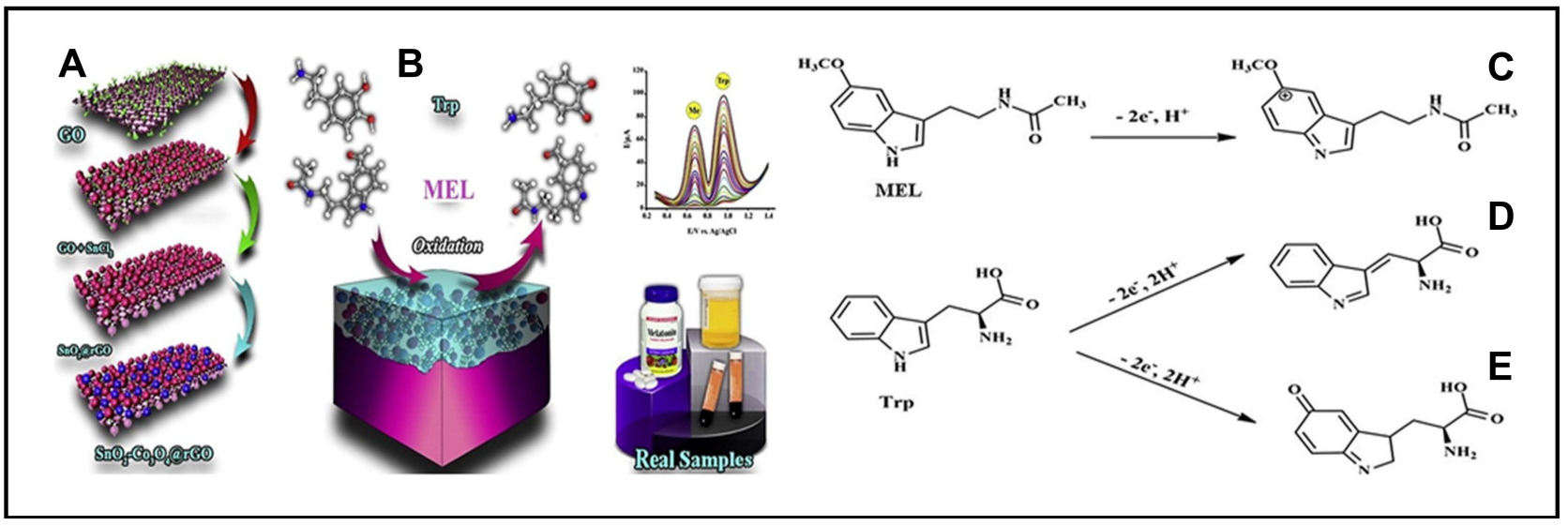

Figure 3 Synthesis of $\mathrm{SnO}_{2}-\mathrm{Co}_{3} \mathrm{O}_{4} @ r G O$ for the detection of melatonin and tryptophan.

Notes: (A) Step-by-step preparation of $\mathrm{SnO}_{2}-\mathrm{Co}_{3} \mathrm{O}_{4} @ r G O$ starting from graphene oxide (GO). (B) Electrochemical detection of melatonin (MEL) and tryptophan (Trp) in real samples. (C) Two-electron and one-proton oxidation of MEL. (D) and (E) two-electron and two-proton oxidation of tryptophan. Adapted from Materials Science and Engineering: C, Vol 7I, Issue 20I7, Zeinali H, Bagheri H, Monsef-Khoshhesab Z, Khoshsafar H, Hajian A, Nanomolar simultaneous determination of tryptophan and melatonin by a new ionic liquid carbon paste electrode modified with $\mathrm{SnO}_{2}-\mathrm{Co}_{3} \mathrm{O}_{4} @ \mathrm{rGO}$ nanocomposite, Pages No. 386-394, Copyright (20I7), with permission from Elsevier. ${ }^{62}$ 
measurements. The detection range was $0.02-6.00 \mu \mathrm{M}$ and the LODs were 4.1 and $3.2 \mathrm{nM}$ for melatonin and tryptophan, respectively; the measurements were taken using both human and pharmaceutical samples. Given that melatonin undergoes a two-electron/one-proton reaction, while tryptophan undergoes a two-electron/two-proton reaction, this method is unlikely to accommodate the detection of serotonin, which is structurally similar to both analytes and their intermediate product in the melatonin biosynthetic pathway, ${ }^{63-65}$ thus limiting the applicability of the $\mathrm{SnO}_{2}-\mathrm{Co}_{3} \mathrm{O}_{4} @ \mathrm{rGO} / \mathrm{IL} / \mathrm{CPE}$ sensor. Furthermore, the synthesis of $\mathrm{SnO}_{2}-\mathrm{Co}_{3} \mathrm{O}_{4} @ \mathrm{rGO} / \mathrm{IL} / \mathrm{CPE}$ is a time-consuming and complex multistep process, thus limiting its widespread use (Figure 3). ${ }^{62}$

\section{DPV-Based Detection of Tryptophan}

It is known that the electrochemical sensing of tryptophan shows sub-optimal performance due to inefficient electron transfer and high overpotential. ${ }^{66}$ Therefore, efforts have been made to develop new materials for the fabrication of modified electrodes to promote electron transfer and reduce overpotential. Carbon nanotubes (CNTs) exhibit attractive characteristics such as high electron transfer between the electroactive species and the electrode surface. In 2018, a modified electrode based on in situ addition of tetrabutylammonium bromide on the $\beta$-cyclodextrin merged with multiwalled CNTs modified GCE (TBABr/ $\beta-C D / M W C N T s /$ GCE) was fabricated to quantitatively analyze the level of tryptophan in the presence of uric acid (UA) and AA. ${ }^{67}$ The DPV measurement demonstrated a linear relationship to the tryptophan concentration of $1.5-30.5 \mu \mathrm{M}$ and the LOD was $0.07 \mu \mathrm{M}$, as well as excellent selectivity, good stability, and reproducibility. The sensitivity and selectivity of tryptophan detection by TBABr/b-CD/MWCNTs/GCE was due to good conductivity of MWCNTs, molecular recognition capability $\beta-\mathrm{CD}$, hydrophobic interaction, and electrostatic attraction of TBABr with tryptophan. The ion of TBABr is $\mathrm{Bu}_{4} \mathrm{~N}^{+}$is large, has low conductivity and high activation enthalpy values of charge transport. ${ }^{68}$ Therefore, using another cationic surfactant such as sodium tetrafluoroborate, ${ }^{69}$ which has a higher ionic conductivity, ${ }^{68}$ can be used to improve the sensitivity of the electrode.

To fabricate a high-performance electrode for tryptophan, investigators utilized graphene (GR); as it outperforms CNTs. ${ }^{70} \mathrm{Li}$ et al ${ }^{71}$ and Haldorai et $\mathrm{al}^{72}$ fabricated electrodes by hybridizing palladium-copper-copper oxide ( $\mathrm{Pd}-\mathrm{Cu}-$ $\left.\mathrm{Cu}_{2} \mathrm{O}\right)$ and tin oxide nanoparticles $\left(\mathrm{SnO}_{2}\right)$, respectively, on rGO. The DPV voltammogram demonstrated a LOD of
$1.9 \mathrm{nM}$ and $0.04 \mu \mathrm{M}$ for $\mathrm{Pd}-\mathrm{Cu}-\mathrm{Cu}_{2} \mathrm{O}$ and $\mathrm{SnO}_{2}$, respectively. Both the groups used the hydrothermal route for the development of tryptophan-sensitive electrodes. The major limitations of the hydrothermal synthesis include the need for expensive autoclaves and the impossibility of observing the crystal growth because of the steel tube of the autoclave. ${ }^{73}$ Also, $\mathrm{Pd}-\mathrm{Cu}-\mathrm{Cu}_{2} \mathrm{O}$ was only applied for the detection of tryptophan in milk and urine samples, thus requires testing in presence of other amino acids.

Generally, two enantiomers of tryptophan (L-tryptophan and D-tryptophan) exist in nature, ${ }^{74}$ but their biological activities are different. ${ }^{75,76}$ L-tryptophan showed a significant impact on the normal functions of life, and its deficiency may cause several diseases. On the other hand, D-tryptophan was identified as a bioactive compound for postembryonic ovarian development in the Dugesia ryukyuensis. ${ }^{77}$ Recently, a nanocomposite was synthesized from rGO, carboxymethyl cellulose (CMC), and polyaniline (PANI), for the selective detection of L- and D-tryptophan by DPV measurements. ${ }^{78}$ The developed nanocomposite was decorated on a GCE to obtain an electrochemical sensor $\mathrm{rGO} / \mathrm{PANI} / \mathrm{CMC} / \mathrm{GCE}$. The sensor showed a linear response range from 0.01 to 5 $\mathrm{mM}$ with a detection limit of $0.07 \mu \mathrm{M}$ and $0.005 \mu \mathrm{M}$ for L-tryptophan and D-tryptophan, respectively. In the absence of strong coordination ions, chiral recognition happens because of the formation of two different diastereoisomeric complexes between enantiomer and selector with different binding energy. This difference in binding energy is reflected in the electrochemical signal to differentiate between the two enantiomers. The rGO/PANI/CMC/GCE has an enantiomeric selectivity of 2.26. Furthermore, chemicals like epinephrine, dopamine, histamine, adrenaline, serotonin, tryptamine, tyramine, and phenethylamine were used for interference analysis. The result exhibited that the existence of these chemicals had a minor influence on electrochemical chiral recognition. The major downside of this sensor is in situ preparation of nanocomposite. The in situ synthesis uses a lot of initial material chemicals and a short period to execute the polymerization process. ${ }^{79}$ Moreover, the unreacted educts of the in situ reaction might influence the properties of the final material, which can change the entire electrochemistry and hamper reproducibility. ${ }^{80}$

\section{DPV-Based Detection of Melatonin}

Gomez et al $^{81}$ detected melatonin and serotonin in pharmaceutical samples. In this study, graphene was utilized as a base material due to its high electrical conductivity and surface area. They used single/multi-walled CNTs to 
modify carbon screen-printed electrodes for their DPV method, which exhibited LODs of $0.4 \mu \mathrm{M}$ for serotonin and $1.1 \mu \mathrm{M}$ for melatonin. The attempt was to perform the simultaneous detection of melatonin and serotonin in biological samples. However, the electrode was not sensitive enough to be applied to biological samples, and can only be applied for pharmaceutical sample analysis.

A platform that employed a simpler, faster nanocomposite production process was later developed, in which self-assembling gold nanoparticles (AuNPs) linked to molybdenum disulfide $\left(\mathrm{MoS}_{2}\right)$ nanoflakes $\left(\mathrm{Au}-\mathrm{MoS}_{2}\right)$ were prepared by probe sonication. The rapid synthesis of $\mathrm{Au}-\mathrm{MoS}_{2}$ composites was performed by sonicating a mixture of $\mathrm{MoS}_{2}$ nanoflakes and AuNPs at 30\% amplitude for $5 \mathrm{~min}$. The Au-MoS $\mathrm{S}_{2}$ composite was drop-casted onto GCEs to create Au-MoS $\mathrm{S}_{2}$-GCE sensing platforms. The Au- $\mathrm{MoS}_{2}$-GCEs exhibited a high surface-to-volume ratio (high catalytic activity) due to a high electrochemically active surface area. This $\mathrm{Au}-\mathrm{MoS}_{2}$-GCE platform was applied to the simultaneous detection of UA and melatonin $^{82}$ and achieved a LOD of $18.2 \mathrm{nM}$ for UA and $15.7 \mathrm{nM}$ for melatonin. Although the $\mathrm{Au}-\mathrm{MoS}_{2}$-GCE sensors generated reproducible results and exhibited good operational and storage stability, the clinical usefulness of this dual $\mathrm{UA} /$ melatonin detection system was not demonstrated in this study, as the samples analyzed were urine samples spiked with UA and melatonin. Quantifying melatonin levels in urine is not common practice in clinical studies, because only small quantities $(\sim 5 \%)$ of endogenous melatonin are present in urine samples; ${ }^{83,84}$ levels of a6MTs in urine samples are typically measured instead, because the concentration of a6MTs in urine is $2-3$ times higher than that of melatonin, and urinal a6MTs concentration reflects that of endogenous melatonin. ${ }^{85-87}$

In 2017, Rajkumar et $\mathrm{al}^{88}$ synthesized highly stable palladium nanoparticles on a porous carbon aerogel $(\mathrm{Pd} / \mathrm{CA})$ using microwave reduction, which they used for the electrochemical sensing of melatonin and dopamine. Due to its high surface area $\left(851.8 \mathrm{~m}^{2} / \mathrm{g}\right)$ and pore volume $\left(3.021 \mathrm{~cm}^{3} / \mathrm{g}\right)$, the $\mathrm{Pd} / \mathrm{CA}$ nanocomposite displayed exceptional electrocatalytic activity and selectivity for both melatonin and dopamine. Various voltammetric methods, such as DPV, LSV, and OSWSV, were applied using the $\mathrm{Pd} / \mathrm{CA}$ electrode to analyze melatonin and dopamine samples in the presence of interfering biomolecules, including AA and UA. The Pd/CA-modified electrode with DPV exhibited impressive linear response ranges for the electrochemical detection of melato$\operatorname{nin}(0.02-500 \mu \mathrm{M})$ and dopamine $(0.01-100 \mu \mathrm{M})$ with LODs of 2.6 and $7.1 \mathrm{nM}$, respectively. The $\mathrm{Pd} / \mathrm{CA}$ nanocomposite was produced using a relatively simple microwave reduction method; however, the synthesis of the carbon aerogel, as well as $\mathrm{Pd} / \mathrm{CA}$ curing, required carbonization procedures to be performed under $\mathrm{N}_{2}$ gas at $900^{\circ} \mathrm{C}$ and $400^{\circ} \mathrm{C}$, respectively. ${ }^{88}$ Efforts should be made to simplify the $\mathrm{Pd} / \mathrm{CA}$ fabrication process to facilitate the scale-up of the electrode.

In 2018, the combined measurement of acetaminophen, epinephrine, and melatonin was performed for the first time, using a DPV method with CPE sensors modified with zinc ferrite nanoparticles $\left(\mathrm{ZnFe}_{2} \mathrm{O}_{4} \mathrm{NPs} / \mathrm{CPE}\right){ }^{89}$ These zinc ferrite nanoparticles have a high surface-tovolume ratio and display good electrocatalytic activity toward melatonin, acetaminophen, and epinephrine. The best electrocatalytic activity was obtained at working voltages of $0.55,0.35$, and $0.09 \mathrm{~V}$, for melatonin, acetaminophen, and epinephrine, respectively. The linear detection ranges and LODs were $6.5-145 \mu \mathrm{M}$ and $3 \mu \mathrm{M}$ for melatonin, $6.5-135 \mu \mathrm{M}$, and $0.4 \mu \mathrm{M}$ for acetaminophen, and 5-100 $\mu \mathrm{M}$ and $0.7 \mu \mathrm{M}$ for epinephrine, respectively; the samples analyzed were spiked human serum samples and pharmaceutical products. Although the $\mathrm{ZnFe}_{2} \mathrm{O}_{4} \mathrm{NPs} / \mathrm{CPE}$ sensor exhibited low interference by other biomolecules, the LOD using $\mathrm{ZnFe}_{2} \mathrm{O}_{4} \mathrm{NPs} / \mathrm{CPE}$ sensors is relatively high, so it is irrelevant for human samples.

In 2019, the same research group that developed the $\mathrm{ZnFe}_{2} \mathrm{O}_{4} \mathrm{NPs} / \mathrm{CPE}$ sensor ${ }^{89}$ developed a more sensitive electrochemical platform to analyze melatonin, dopamine, and acetaminophen simultaneously. ${ }^{90}$ The DPV was applied on the samples by using CPE sensors coated with aluminum oxide-supported palladium nanoparticles $\left(\mathrm{PdNP} / \mathrm{Al}_{2} \mathrm{O}_{3}\right.$ ), exhibiting ranges of detection for dopamine, acetaminophen, and melatonin of $50 \mathrm{nM}-1.45$ $\mathrm{mM}, 40 \mathrm{nM}-1.4 \mathrm{mM}$, and $6.0 \mathrm{nM}-1.4 \mathrm{mM}$, respectively. The LOD was $36.5 \mathrm{nM}$ for dopamine, $36.5 \mathrm{nM}$ for acetaminophen, and $21.6 \mathrm{nM}$ for melatonin.

The synthesis of the $\mathrm{ZnFe}{ }_{2} \mathrm{O}_{4} \mathrm{NPs} / \mathrm{CPE}$ and $\mathrm{PdNP} / \mathrm{Al}_{2} \mathrm{O}_{3}$ electrodes, especially the paste preparation method was laborious; this poses future challenges for scale-up and mass production. Moreover, the use of Pd increased the cost of electrode production, thus preventing its public utilization. Therefore, research groups have investigated the replacement of expensive metals for cheaper alternatives to produce melatonin sensors. In the same vein, a highperformance melatonin sensor was produced by decorating inexpensive carbon nanofibers (CNFs) with $\mathrm{FeCo}$ bimetallic alloys and then depositing them on GCEs (FeCo@CNFs/ GCE). ${ }^{91}$ FeCo displayed high electrocatalytic and electron 
transfer activities, which enabled rapid and continuous melatonin analysis. Pharmaceutical samples containing melatonin were analyzed with the FeCo@CNFs/GCEs, exhibiting a detection limit of $2.7 \mathrm{nM}$; this detection limit is sufficient for analyzing pharmaceutical samples. Similar to the other electrochemical sensors discussed above, the key limitation of the FeCo@CNFs/GCE sensor is its long and complex fabrication process; the synthesis of FeCo@CNFs alone takes $24 \mathrm{~h}$. Additionally, nanofiber stabilization takes $1 \mathrm{~h}$ at $280^{\circ} \mathrm{C}$, followed by carbonization at $800^{\circ} \mathrm{C}$ for $2 \mathrm{~h}$. A short and simple process should be developed to produce bimetal nanowires.

Recently, a sensitive DPV melatonin sensor based on integrated 2D materials was developed by Liu et al. ${ }^{92}$ Electrode of melatonin sensor was fabricated by decorating a ternary complex of Tin disulfide $\left(\mathrm{SnS}_{2}\right)$ nanoflakes, $\mathrm{GO}$, and $\beta-\mathrm{CD}$ on the surface of SPEs $\left(\mathrm{SnS}_{2} / \mathrm{GO} / \beta-\mathrm{CD} /\right.$ SPE). ${ }^{92}$ The DPV method and $\mathrm{SnS}_{2} / \mathrm{GO} / \beta-\mathrm{CD} / \mathrm{SPE}$ sensor combination had a linear range of melatonin detection from $1 \mathrm{nM}$ to $100 \mu \mathrm{M}$, with a LOD of $0.17 \mathrm{nM}$. The $\mathrm{SnS}_{2} / \mathrm{GO} / \beta-\mathrm{CD} / \mathrm{SPE}$ electrochemical sensor was successfully applied to detect melatonin in pharmaceutical samples and human saliva, both of which correlated well with corresponding ELISA results. The $\mathrm{SnS}_{2} / \mathrm{GO} / \beta-\mathrm{CD} / \mathrm{SPE}$ sensor had a fast response time, was made of inexpensive tin and graphene materials, and was relatively easy to operate, thus overcoming the limitations typically found in conventional melatonin sensors. The sensitivity of the $\mathrm{SnS}_{2} / \mathrm{GO} / \beta-\mathrm{CD} / \mathrm{SPE}$ sensor is high enough to analyze nocturnal biological samples; however, its sensitivity must be enhanced to extend its applicability to daytime samples. Additionally, the fabrication time of $\mathrm{SnS}_{2} / \mathrm{GO} / \beta$ $\mathrm{CD} / \mathrm{SPE}$ sensors is almost $24 \mathrm{~h}$ and includes hydrothermal synthesis at $200^{\circ} \mathrm{C}$ for $12 \mathrm{~h}$, which highlights another potential area for improvement.

In plants, melatonin is a potent antioxidant and growth promoter, protecting against oxidative stress and facilitating adaptation to harsh environmental conditions. ${ }^{92}$ Plants operate under a wide range of $\mathrm{pH}$ conditions, making it difficult to develop electrochemical sensors to monitor plant bioactive molecules in vivo. ${ }^{93}$ To tackle this issue, a DPV system using a copper oxide ( $\mathrm{CuO})$-poly(L-lysine)/ graphene electrode as a sensor was developed to assess melatonin and vitamin B6 (pyridoxine, PN) levels in plants in situ. ${ }^{94}$ Melatonin and PN detection was dependent on $\mathrm{CuO}$ and poly(L-lysine), while the conductivity of the 3D-graphene layer amplified the catalytic current. Between $\mathrm{pH}$ 6.8-7.4, the current-time curve displayed a detection range of $0.016-1110 \mu \mathrm{M}$ for melatonin, with a LOD of $12 \mathrm{nM}$. The linear detection range for PN was 3 $-2076 \mu \mathrm{M}$ and the LOD was $2.3 \mu \mathrm{M}$. Because the $\mathrm{pH}$ of fruit varies according to species and level of maturity, further research is warranted to improve the $\mathrm{pH}$ range of working electrodes.

\section{SWV-Based Detection of Tryptophan}

SWV is a fast voltammetric technique that is routinely applied for quantitative analyses. Although SWV was first reported as long ago as $1957,{ }^{95}$ its practical use has been limited by the level of available technology. Recent advancements in both analog and digital electronics have made the incorporation of SWV into modern polarographic analyzers feasible. SWV is much faster than normal pulse or DPV; DPV generally exhibits scan rates of $1-10 \mathrm{mV} / \mathrm{s}$, whereas the scan rates of SWV are typically $>1 \mathrm{~V} / \mathrm{s}^{96,97}$

Owing to advantages including low background current (compared with solid graphite or other noble metal electrodes), easy synthesis, the feasibility of incorporating various substances during the paste preparation, large potential window, inexpensive, straightforward surface renewal process, and ease of miniaturization, the CPEs are widely applicable in both electrochemical studies and electroanalysis. ${ }^{98-102}$ In 2015, Beitollahi et al ${ }^{103}$ modified the $\mathrm{CPE}$ with 2-chlorobenzoylferrocene/Ag- $\mathrm{ZnO}$ nanoplates (2CBFAGZCPE) for the detection of tryptophan along with captopril. The SWV peak current increased linearly with tryptophan concentration in the range of 0.05 to $20.0 \mu \mathrm{M}$ and a detection limit of $0.02 \mu \mathrm{M}$ was obtained. The electrochemical analysis at the surface of modified electrode 2CBFAGZCPE exhibited that electrooxidation is catalyzed at $\mathrm{pH}$ 7.0. This result indicated that the fabricated sensor 2CBFAGZCPE might be $\mathrm{pH}$ sensitive, which will limit its applicability for the detection of tryptophan in various patient samples such as in acidosis, acute pancreatitis, and poisoning, etc. ${ }^{104-106}$ Though the application of transition metal complexes (eg, $\mathrm{Ag}-\mathrm{ZnO}$ ) for the development of tryptophan sensor has shown electrocatalytic properties, the linear range of tryptophan detection by 2CBFAGZCPE was low because the electron transfer process is slow and the overpotential is high for the direct oxidation of tryptophan on the CPE. ${ }^{107,108}$

In 2016, nickel oxide $(\mathrm{NiO})$ and copper oxide $(\mathrm{CuO})$ nanoparticles and modified GR were decorated on GCE to fabricate a sensor for simultaneous determination of tryptophan, dopamine, and acetaminophen using square wave 
voltammetry. ${ }^{109}$ The GCE is better than CPE in terms of promoting electron transfer and thereby enhancing sensitivity. ${ }^{110}$ GR was modified by using citric acid to produce more functional groups, which benefits the deposition of dispersed metal particles. $\mathrm{NiO}-\mathrm{CuO} / \mathrm{GR}$ modified electrode was prepared by electro-deposition. Afterwards, a modified electrode was prepared by electro-deposition of $\mathrm{NiO}-\mathrm{CuO}$ and citrate modified GR on GCE (NiO-CuO/GR/ $\mathrm{GCE}$ ). The $\mathrm{NiO}-\mathrm{CuO} / \mathrm{GR} / \mathrm{GCE}$ electrode displayed that the linear response ranges for detecting tryptophan, dopamine, and acetaminophen were $0.3-40 \mu \mathrm{M}, 0.5-20 \mu \mathrm{M}$, and 4- $400 \mu \mathrm{M}$, respectively, and the detection limits were 0.1 $\mu \mathrm{M}, 0.17 \mu \mathrm{M}$, and $1.33 \mu \mathrm{M}$. Similarly, He et $\mathrm{al}^{111}$ used GCE and modified it with a nanocomposite of cuprous oxide and $\mathrm{rGO}\left(\mathrm{Cu}_{2} \mathrm{O}-\mathrm{rGO} / \mathrm{GCE}\right)$. The cuprous oxide copper oxides are p-type semiconductors with better ability to promote electron transfer process, are inexpensive, comparatively lower toxicity, and effective antimicrobial activity. ${ }^{112-115}$ The oxidation peak current of tryptophan exhibited a slightly better linear relationship with its concentration in the range of $0.02-20 \mu \mathrm{M}$, and the LOD was $0.01 \mu \mathrm{M}$, as analyzed by SWV. The tryptophan sensor $\mathrm{Cu}_{2} \mathrm{O}-\mathrm{rGO} / \mathrm{GCE}$ was applied for the quantification of tryptophan in human serum samples and commercial amino acid injections. With a high recovery rate of $97.0-102.3 \%$, the authors showed that the sensor can be used for the determination of tryptophan in real samples such as human serum. However, the $\mathrm{Cu}_{2} \mathrm{O}-\mathrm{rGO} / \mathrm{GCE}$ sensor used sulfuric acid as a supporting electrolyte for the detection of tryptophan, which is a very strong and highly corrosive acid. Sulfuric acid can decompose lipids and proteins, by ester and amide hydrolysis upon contact with living tissues, like skin or flesh. The sulfuric acid exhibits a strong dehydrating property on carbohydrates, liberating extra heat leading to secondary thermal injuries. ${ }^{116,117}$ The use of sulfuric acid as a supporting electrolyte for the tryptophan detection limits the practical applicability of $\mathrm{Cu}_{2} \mathrm{O}-\mathrm{rGO} /$ GCE. In addition, the LOD can be further improved by the modification of metal oxide on the GCE.

In 2021, GCE was modified with a nanocomposite of $\mathrm{CuO}$ nanoflakes anchored with polythiophene (PT-CuO/ GCE). ${ }^{118}$ Despite the aforementioned exciting features of copper oxides in sensing, it has few limitations such as low conductivity and aggregation. To improve these shortcomings, a CuO-PT nanocomposite was prepared. Polythiophene, an exceptional organic conducting polymer, can proficiently transfer the electric charge produced by the biochemical reaction to the electronic circuit. The use of PT-CuO/GCE electrode does not show enhancement in LOD of $15 \mathrm{nM}$ and linear detection range of $0.1 \mu \mathrm{M}$ to $1 \mathrm{mM}$ for tryptophan as compared with previous reports. Further research is needed to explore novel materials used in the eco-friendly and sensitive detection of tryptophan.

\section{SWV-Based Detection of Melatonin}

Several SWV-based sensing platforms have been developed for the detection of melatonin and other related biomolecules. Molaakbari et $\mathrm{al}^{119}$ fabricated zinc oxide ( $\mathrm{ZnO})$ nanorods to coat $\mathrm{CPEs}(\mathrm{ZnO} / \mathrm{CPE})$, thus generating sensors for the simultaneous evaluation of dopamine, melatonin, methionine, and caffeine levels. ${ }^{119}$ The combined SWV-ZnO/CPE technique had LODs of $5.0 \mu \mathrm{M}, 750.0$ $\mu \mathrm{M}, 700.0 \mu \mathrm{M}$, and $450.0 \mu \mathrm{M}$ for dopamine, melatonin, methionine, and caffeine, respectively; the linear detection range was $0.3-0.1 \mathrm{mM}$. The sensitivity for dopamine detection using $\mathrm{ZnO} / \mathrm{CPEs}$ may be adequate for use; however, the LOD for melatonin was relatively poor. Therefore, $\mathrm{ZnO} / \mathrm{CPEs}$ cannot be applied for melatonin detection in most biological fluids.

The electrocatalytic activity of chemically modified electrodes depends on the electrode materials. To improve sensitivity, CPEs were modified using graphene decorated with magnetite $\left(\mathrm{Fe}_{3} \mathrm{O}_{4}\right)$ magnetic nanoparticles $\left(\mathrm{G} / \mathrm{Fe}_{3} \mathrm{O}_{4} /\right.$ CPE), then applied with SWV to simultaneously detect melatonin and dopamine. ${ }^{120}$ The synergistic action of graphene and the $\mathrm{Fe}_{3} \mathrm{O}_{4}$ magnetic nanoparticles created a large surface area, as well as good electrical conductivity and electrocatalysis. The $\mathrm{G} / \mathrm{Fe}_{3} \mathrm{O}_{4} / \mathrm{CPE}$ displayed a broader linear range and increased LOD compared to the $\mathrm{ZnO} / \mathrm{CPE}$ : the range of detection was the same for both melatonin and dopamine $(0.02-5.80 \mu \mathrm{M})$, but the LOD differed for each (8.40 and $6.50 \mathrm{nM}$ for melatonin and dopamine, respectively). Although the sensitivity of G/ $\mathrm{Fe}_{3} \mathrm{O}_{4} / \mathrm{CPEs}$ was higher than that of $\mathrm{ZnO} / \mathrm{CPEs}, \mathrm{G} /$ $\mathrm{Fe}_{3} \mathrm{O}_{4} / \mathrm{CPEs}$ displayed poor molecular specificity. A 12fold excess of tryptophan seriously interfered with the melatonin measurements taken using $\mathrm{G} / \mathrm{Fe}_{3} \mathrm{O}_{4} / \mathrm{CPEs}$. Under standard physiological conditions, tryptophan levels in human beings are more than 1000-fold higher than melatonin and can be higher in certain disease conditions such as schizophrenia. ${ }^{121-123}$ Tryptophan interference is therefore an important limitation of the $\mathrm{G} / \mathrm{Fe}_{3} \mathrm{O}_{4} / \mathrm{CPE}$ sensor.

In another investigation, the simultaneous detection of melatonin and serotonin was achieved by combining SWV with an electrochemical sensor, which was fabricated by 
depositing acetylene black-chitosan nanoparticles (AB-C) onto gold electrodes (AB-C/GEs). ${ }^{124}$ The authors of this study claimed that the biopolymer-based sensor was economical; nonetheless, the use of gold electrodes nullifies this assertion. The LOD using the AB-C/GEs was $0.16 \mu \mathrm{M}$ for serotonin and $1.9 \mu \mathrm{M}$ for melatonin. The $\mathrm{AB}-\mathrm{C} / \mathrm{GE}$ sensors were able to detect melatonin in the presence of equimolar concentrations of dopamine and AA. More sensors should be developed using biologically derived, economical compounds such as chitosan, agar, and cellulose but using cheaper electrodes like CPEs.

In 2019, a sensitive and selective electrochemical sensor was developed for the simultaneous sensing of melatonin, norepinephrine, and nicotine. The sensor comprised nicotinamide adenine dinucleotide (NAD) immobilized on gamma $(\gamma)$-irradiated tungsten trioxide nanoparticles $\left(\mathrm{WO}_{3}\right)$, which was used to coat a GCE (Figure 4). ${ }^{125}$ The nicotinamide coenzyme is required to catalyze the reversible $\mathrm{NAD}^{+}$to NADH redox reaction, which is a major source of energy production during cellular respiration. ${ }^{126}$ The strong redox properties of the deposited NAD enabled the differential electro-oxidation of melatonin, norepinephrine, and nicotine, thus enabling the identification and quantification of the three analytes. SWV was applied due to its suitability, selectivity, and sensitivity; this technique facilitated the electrocatalytic analysis of melatonin, norepinephrine, and nicotine in the presence of various biomolecules that would potentially cause interference in in vivo samples, such as dopamine, serotonin, epinephrine, cotinine, adenosine diphosphate, adenosine triphosphate, glucose, folic acid, tyrosine, tryptophan, UA, cysteine, and AA. ${ }^{125}$ The LODs for melatonin, norepinephrine, and nicotine were $2.6 \mathrm{nM}, 1.4 \mathrm{nM}$, and $1.7 \mathrm{nM}$, respectively. In the same study, sensitive GCEs modified with $\gamma$ ray-irradiated ethylenediaminetetraacetic acid- $\mathrm{WO}_{3}$ nanoparticles harboring immobilized NAD were developed for melatonin detection. However, the use of $\gamma$ rays in electrode fabrication is a major concern due to their toxicity (Figure 4A); short exposure to $\gamma$ rays readily causes mutations in DNA, leading to tumor formation or cancer. ${ }^{127}$ Therefore, biologically and environmentally benign methods should be developed to fabricate electrochemical sensors.

In response to health and environmental concerns associated with electrode manufacturing, electrode fabricated from the biodegradable and biocompatible waterproof

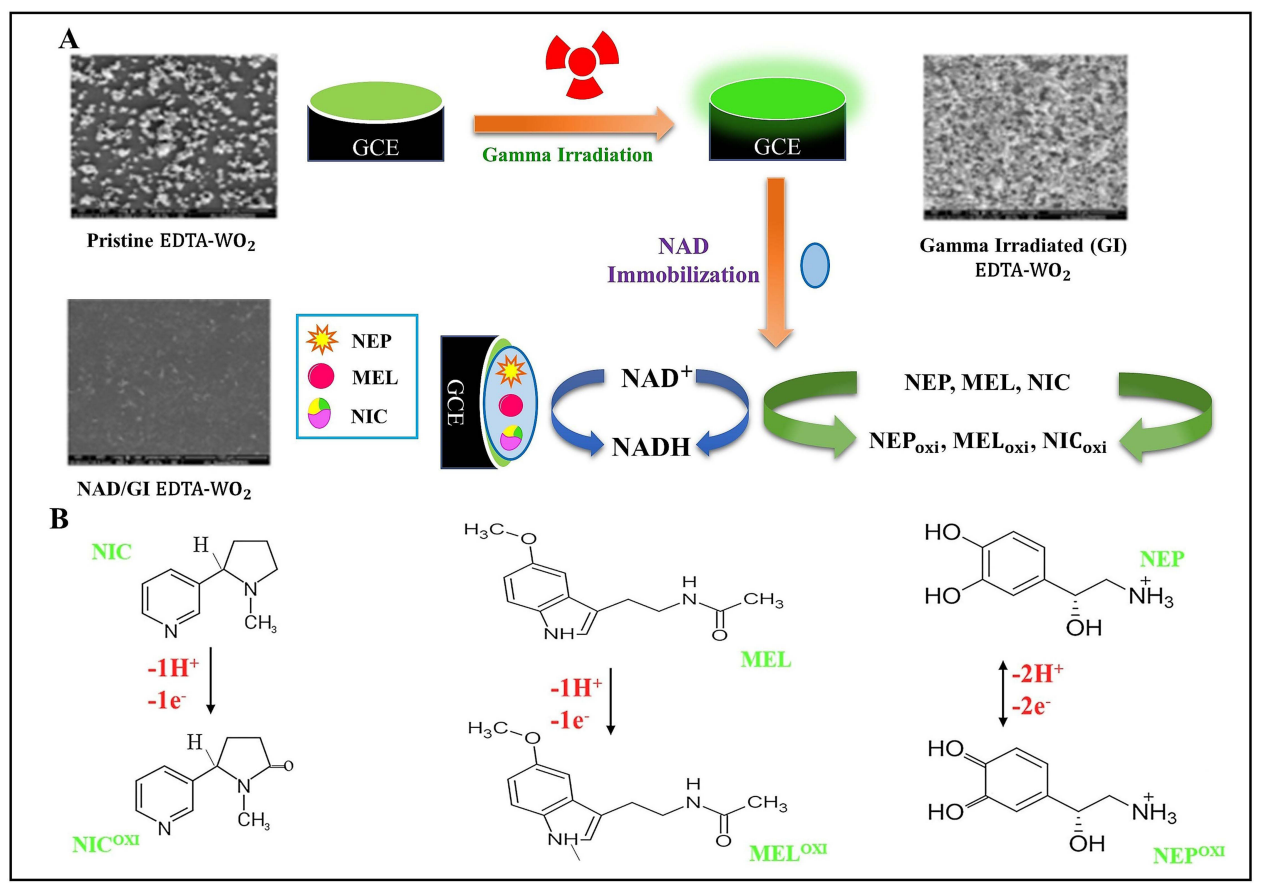

Figure 4 Synthesis and detection of nicotine, melatonin, and norepinephrine by NAD/GI EDTA-WO 3 /GCE.

Notes: (A) Gamma irradiation mediated immobilization of nicotinamide adenine dinucleotide (NAD) on glassy carbon electrodes (GCEs). (B) Mechanism of the electrochemical determination of nicotine (NIC) and melatonin (MEL) by one proton and one electron oxidation, and of norepinephrine (NEP) by two protons and twoelectron oxidations. Adapted from Biosensors and Bioelectronics, Vol 143, Issue 2019, Anithaa AC, Asokan K, Lavanya N, Sekar C, Nicotinamide adenine dinucleotide immobilized tungsten trioxide nanoparticles for simultaneous sensing of norepinephrine, melatonin and nicotine, Page No. III598, Copyright (20I9), with permission from Elsevier. $^{125}$

Abbreviations: EDTA, ethylene diamine tetraacetic acid; GI, gamma irradiation; $\mathrm{WO}_{3}$, tungsten trioxide nanoparticles. 
paper was developed, generating a disposable electrochemical sensing platform for the quantitative and qualitative assessment of melatonin and paracetamol. ${ }^{128}$ To achieve this, a conductive ink was synthesized using graphite powder dispersed in nail polish (GPT), which was then applied to the waterproof paper using a screen-printing technique, thus generating GPT/waterproof paper electrode (GPT/WPEs) (Multimedia Component 1). The reference electrodes were modified with silver ink. A method combining SWV with the GPT/WPEs was applied to analyze melatonin samples, demonstrating a linear detection range of $0.80-100 \mu \mathrm{M}$ and a LOD of $32.5 \mathrm{nM}$. The SWVGPT/WPE method was then used to detect paracetamol and melatonin in synthetic saliva, sweat, and urine samples. Although this waterproof paper-based electrochemical device is straightforward, disposable, and costeffective, it is yet to be tested with real biological samples and for tryptophan interference. ${ }^{120}$ Furthermore, the range and limit of detection of the GPT/WPE should be improved before it can be used on a mass scale. Other conducting polymers such as poly(3,4-ethylenedioxythiophene) and poly(styrenesulfonate) exhibit higher conductivities than graphite, and could therefore be used to further enhance sensitivity and LOD.

As discussed above, the specific detection of melatonin in complex biological samples is a significant challenge. Some recently developed methods displayed good selectivity but compromised on the LOD and detection range, whereas others were not tested for molecular interference with real biological samples. Fabricating molecular recognition materials for voltammetric analyses could increase the specificity of electrodes. Molecular imprinting technology is a tool for synthesizing robust molecular recognition materials for the detection of molecules such as hormones, proteins, nucleotides, drugs, pollutants, and food. The principle of MIT relies on the formation of complexes between an analyte, which acts as a template, and a functional monomer. In the presence of a cross-linking agent, a three-dimensional polymer network is formed between the analyte and the monomer. Following polymerization, the analyte (template) is stripped from the polymer, leaving specific recognition sites that are complementary to the analyte in size, shape, and chemical functionality (Figure 5). ${ }^{129}$ Molecular recognition typically occurs via intermolecular interactions (eg, hydrogen bonds,

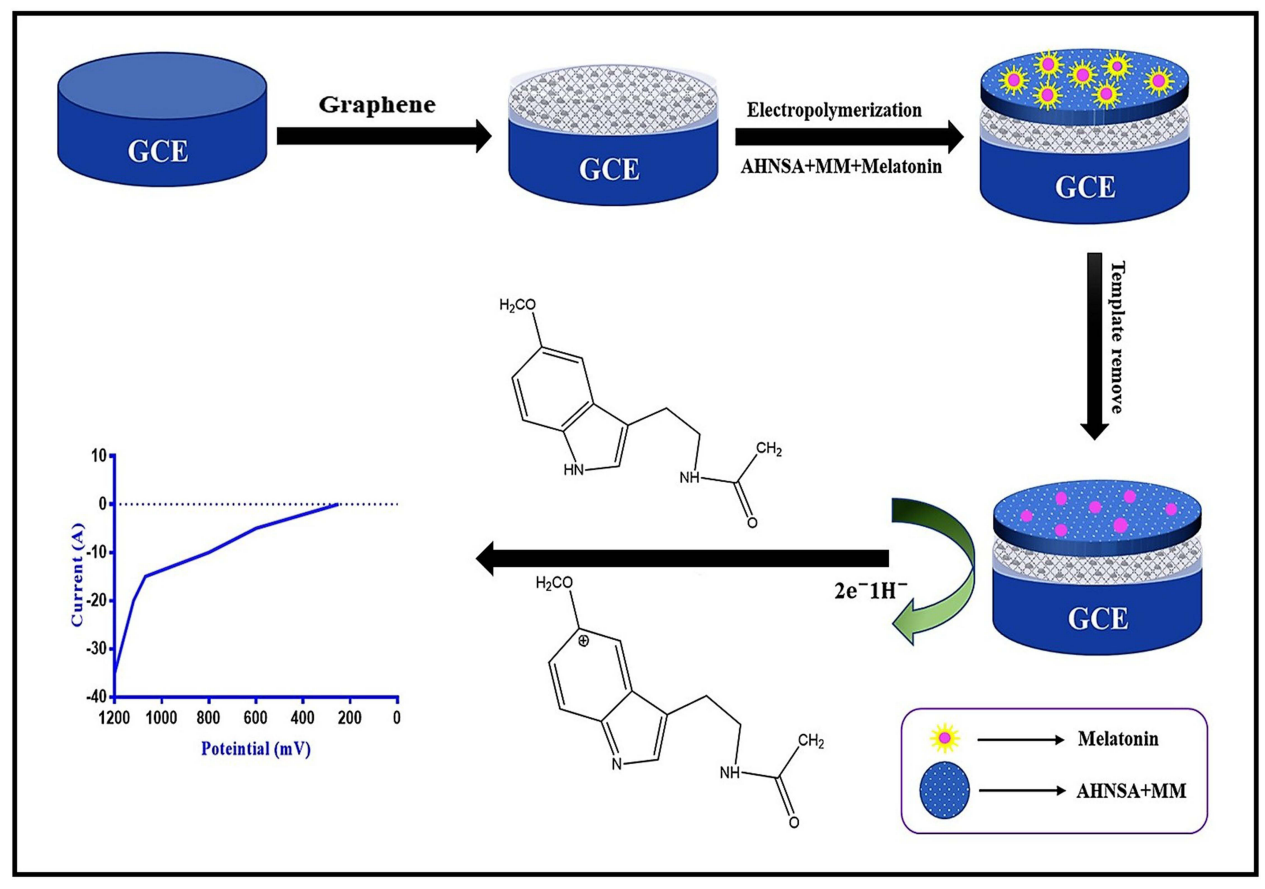

Figure 5 Scheme showing the fabrication of a molecularly imprinted melatonin sensor using a graphene and co-polymer composite.

Notes: The graphene was dropped on the surface of GCEs and dried at room temperature overnight. The film containing the imprinted copolymer was prepared by electropolymerization. The template was then removed to obtain the molecularly imprinted surface for the detection of melatonin. Adapted with permission of Royal Society of Chemistry, from Graphene and Co-polymer composite based molecularly imprinted sensor for ultratrace determination of melatonin in human biological fluids, Gupta P, Goyal RN, RSC Advances, Vol 5, edition 50, Copyright (2015); permission conveyed through Copyright Clearance Center, Inc. ${ }^{130}$

Abbreviations: GCE, glassy carbon electrode; AHNSA, 4-amino-3-hydroxy-I-naphthalene sulfonic acid; MM, melamine. 
ionic bonds, and dipole-dipole interactions) between the analyte molecule and functional groups present in the polymer matrix. Thus, the resulting molecular pattern embedded within the polymer can selectively recognize and bind to the desired analyte. MIT has been successfully applied to modify electrodes for electrochemical sensing. This method offers the advantage of being relatively stable during storage and exhibiting low denaturation rates; instability and denaturation are the primary limitations of biological recognition elements such as enzymes and antibodies.

Recently, an electrochemical microfluidic chip was developed for melatonin detection using MIT. A composite containing graphene, a co-polymer of 4-amino-3hydroxy-1-naphthalenesulfonic acid (AHNSA), and melamine was used to fabricate a novel molecularly imprinted polymer (MIP) sensor to detect melatonin in human serum and urine samples (Figure 5). ${ }^{130}$ The MIP film was produced by depositing graphene onto GCE surfaces and subsequently electropolymerizing AHNSA and melamine in the presence of melatonin. To release the imprinted melatonin molecules from the composite, the modified electrode was cycled between -1.0 and $+1.0 \mathrm{~V}$ at a scan rate of $100 \mathrm{mV} / \mathrm{s}$ for 25 cycles in $0.5 \mathrm{M}$ sulfuric acid. SWV analysis using the MIP sensor displayed a melatonin detection range of $0.05-100 \mu \mathrm{M}$ with a LOD of $60 \mathrm{pM}$.

MIT is a promising tool that could soon replace the use of biomolecules (eg, antibodies, aptamers, and enzymes) for molecular sensing. The selection of a suitable material and preparation protocol is critical for the production of effective MIPs. ${ }^{131}$ However, cost-effective materials to develop MIT-based sensors are currently lacking. ${ }^{132}$ Furthermore, MIT technologies utilize free-radical polymerization and sol-gel processes. Free-radical polymerization involves multiple mechanical grinding and sieving steps to obtain minute particles, which can lead to lower than expected binding affinities. ${ }^{131}$ Moreover, free-radical polymerization can only be performed in bulk, hence requiring a large amount of template material, which renders the process expensive.

\section{Amperometry-Based Detection of Tryptophan}

Amperometry is an electrochemical technique in which current is measured as a function of an independent variable like electrode potential or time. Han et al $^{133}$ pyrolyzed Dandelion pappus and drop-casted on GCE to develop an economical nanoporous carbon sensor for the detection of tryptophan. Owing to the porous structure and large specific surface of nanoporous carbon, it showed an excellent electrocatalytic activity toward tryptophan oxidation with a reduced overpotential and improved current response. The amperometric response of the nanoporous carbon electrode-based sensor was linear in the range of $1 \mu \mathrm{M}$ to $10 \mathrm{mM}$ and the LOD was $0.5 \mu \mathrm{M}$. Tryptophan content in compound amino acid injection and fetal calf serum was assessed by this nanoporous carbon electrode-based sensor and showed a good correlation of 98.17 to $103.93 \%$. The nanoporous carbon electrode sensor is biodegradable and economical, but the drop-casting method used to fabricate this sensor has considerable limitations like the coffee ring effect (CRE). The CRE was observed and interpreted by Deegan et al, ${ }^{134}$ who found that the periphery of the ring was seen to be concentrated with the non-volatile solute particles in contrast to the center of the stain. Meaningful voltammetry results require the formation of uniformly modified surfaces, but drop-casting causes CRE, ${ }^{135}$ which significantly limits the reproducibility of the dropcasted surfaces. A practical approach to mitigate CRE is to explore a variety of solvents in combination with nanoporous carbon electrodes followed by microscopic imaging of the casted surfaces. ${ }^{135}$ The combination which offers minimum CRE should be used to avoid reproducibility issues.

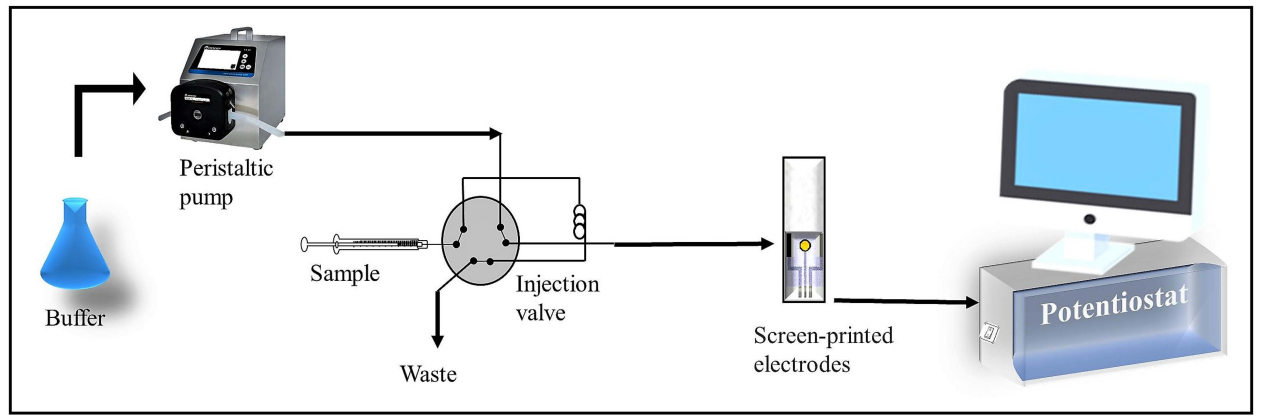

Figure 6 Schematic representation of the simple flow injection analysis system for electrochemical detection. 
A major focus of present-day electroanalytical research is to combine amperometric detection with analysis in a liquid flow. This combination will ensure an enhanced sensitivity, selectivity along with an increase in the throughput of analysis (Figure 6). ${ }^{136}$ Apart from miniaturization and automation, flow injection decreases the volume of reagents, samples, and wastage. Also, flowinjection enhances the diffusion of the sample into the stream of reagent leading to high sensitivity. ${ }^{137}$ Shaidarova et $\mathrm{al}^{138}$ developed a flow-injection-based amperometric sensing platform by electrodepositing AuNPs on a screen-printed electrode for the determination of tryptophan. The dependence of the analytical signal on the concentrations of tryptophan and pyridoxine on the logarithmic coordinates is linear in the ranges from 0.5 $\mu \mathrm{M}$ to $5 \mathrm{mM}$. The use of the amperometric response of an $\mathrm{Au}-\mathrm{SPE}$ electrode under flow injection analysis showed a theoretical throughput of up to 180 samples $/ \mathrm{h}$. Surprisingly, the authors did not report the LOD. Moreover, the flow injection was not fully utilized, for instance, samples were manually prepared by dissolving their precisely weighed portions in a supporting electrolyte solution. A fully automated flow system could be generated by coupling microfluidic channels such as tree-shaped micromixers or serial lamination mixers for the automation of tryptophan mixing and distribution in multiple test chambers. ${ }^{139,140}$

\section{Amperometry-Based Detection of Melatonin}

In 2015, Gomez et $\mathrm{al}^{141}$ developed a single-wall carbon nanotube press-transferred electrode (SW/PTE) to analyze melatonin, tryptophan, and serotonin using a microchip electrophoresis platform. Microchip electrophoresis platforms enable analytic work to be performed rapidly with low sample and reagent volumes and minimum wastage. ${ }^{142,143}$ Furthermore, coupling electrochemical detection with microchip electrophoresis enables quick analyses, high sensitivity, and miniaturization without compromising on performance. By combining SW/PTEs with microchip electrophoresis and amperometric detection, melatonin, tryptophan, and serotonin were rapidly detected in $<150 \mathrm{~s}$ with LODs of 4,1 and 5 $\mu \mathrm{M}$, respectively. The linear ranges of detection were 50-500 $\mu \mathrm{M}$ for both melatonin and tryptophan and 10-200 $\mu \mathrm{M}$ for serotonin. Although this study was promising, analyte separation had to be performed before sample analysis, which potentially interfered with the results. In addition,
Gomez et $\mathrm{al}^{141}$ claimed that their device was portable (as a result of miniaturization) and that their electrodes were disposable. However, the durability and accuracy of their device were not specified; for novel microchip electrophoresisbased detection methods, the number of times the analysis can be performed before the chip requires replacement should be reported. ${ }^{144}$

\section{Stochastic Sensors for Tryptophan Detection}

Stochastic sensors have recently generated considerable interest as they can provide rapid, sensitive, and reconfigurable multi-analyte detection. Stochastic sensors are well known for their capacity of performing both qualitative and quantitative investigation of materials of interest in complex samples. ${ }^{145,146}$ Stochastic sensors function by measuring the ionic transport of analytes through a nanometer-sized pore set within an insulating membrane. ${ }^{147}$ Stochastic sensors are based on several parallel, time-resolved measurements intended to detect, identify and count discrete macromolecular events instead of reading out an average response. Mihai et $\mathrm{al}^{148}$ fabricated four stochastic microsensors with modified graphene materials such as $\beta-\mathrm{CD}$ and nitrogen-doped graphene ( $\beta$-CD/nGR), 2,2-diphenyl-1-picrylhydrazyl and exfoliated graphene (DPPH/exfGR), protoporphyrin IX and nGR (PIX/nGR), and PIX-thermally treated nGR (PIX-nGR-TT) and used for enantioanalysis of tryptophan in whole blood samples. The principle of current development for stochastic sensors is based on channel conductivity. The current development takes place in two steps: the first step when the molecule enters the channel/pore, blocking it absolutely or mostly (the current drops to a zero value), and the second stage where the chirality-dependent binding and redox reaction occurs, leading to the enantioselective determination of tryptophan. The novelty of the screening method is that both enantiomers of tryptophan can be simultaneously examined using the stochastic sensors without pre-processing the whole blood samples. For L-tryptophan, the linear detection range and LOD with $\beta-\mathrm{CD} / \mathrm{nGR}$, DPPH/exfGR, PIX/nGR, and PIX-nGR-TT sensors were $10 \mathrm{fM}-1 \mathrm{mM}$ and $10 \mathrm{fM}, 1$ $\mathrm{fM}-0.10 \mathrm{mM}$ and $1 \mathrm{fM}, 100 \mathrm{fM}-0.10 \mathrm{mM}$ and $100 \mathrm{fM}, 0.10$ $\mathrm{nM}-0.10 \mathrm{mM}$ and $0.10 \mathrm{nM}$, respectively. Similarly, for D-tryptophan, linear detection range and LOD with $\beta-\mathrm{CD} /$ nGR, DPPH/exfGR, PIX/nGR, and PIX-nGR-TT sensors were $1 \mathrm{pM}-\mathrm{nM}$ and $1 \mathrm{pM}, 1 \mathrm{pM}-1 \mathrm{mM}$ and $1 \mathrm{pM}, 0.10$ $\mathrm{pM}-1 \mathrm{mM}$ and $0.10 \mathrm{pM}, 1 \mathrm{pM}-1 \mathrm{nM}$ and $1 \mathrm{pM}$, respectively. Highly sensitive, and reliable results were obtained when the 
developed stochastic sensors were used for the enantioanalysis of tryptophan in blood samples. One drawback of stochastic sensors is poor durability, which restricts their use in the laboratory. ${ }^{149}$ Characteristically, the stochastic microsensors are only a few hundred microns in diameter, and the deposited nanoparticle/electrodes usually rupture or leak current after a few hours of use. In the future, stochastic microsensors should be combined with microfluidics to develop durable lab-on-a-chip technology.

\section{Stochastic Sensors for Melatonin Detection}

Recently, Staden et al ${ }^{150}$ designed two stochastic sensors: one was modified with rGO decorated with titanium oxide $\left(\mathrm{rGO}-\mathrm{TiO}_{2}\right)$ and the other with graphene decorated with $\mathrm{TiO}_{2}$ and AuNPs $\left(\mathrm{rGO} / \mathrm{TiO}_{2} / \mathrm{AuNP}\right)$; both were mixed with a complex of protoporphyrin IX and cobalt. ${ }^{150}$ Both sensors were used to detect melatonin in pharmaceutical products and biological fluids such as breast milk and whole blood; ${ }^{150}$ both displayed the same linear range (9.98 aM-0.99 pM and LOD $9.98 \mathrm{aM}$ ). Even in complex samples, the LODs of the stochastic sensors were excellent, thus demonstrating their applicability for biological sample testing. The major limitations of the melatonin stochastic sensors were the complex fabrication processes and poor reproducibility due to difficulties in generating identical pore sizes.

\section{Optical Detection of Tryptophan and Melatonin}

Optical sensors are a suitable platform to monitor several diseases associated parameters in a personalized fashion. Optical sensors have the potential to completely revolutionize disease diagnosis and treatment. Colorimetric/fluorescence readouts are particularly exciting for sensing applications as the shift in sample color or change in intensity of emitted light can be quantitatively linked to the concentration of a target analyte. Over the last 6 years, fewer novel optical detection methods for tryptophan and melatonin have been reported compared to electrochemical methods.

\section{Optical Detection of Tryptophan}

A simple colorimetric method for the quantitative determination of free tryptophan with 96-well-plate-level throughput was developed. Wu et $\mathrm{al}^{151}$ used purified tryptophanases to enzymatically convert tryptophan to indole (Figure 7). This indole is further reacted with hydroxylamine to form a pink color product with absorption maxima at $530 \mathrm{~nm}$ (Figure 7). ${ }^{151}$ The pink color formation is proportional to the amount of tryptophan in the sample, thereby, quantitative results can be obtained by simple spectrophotometry in two hours. This indolehydroxylamine tryptophan sensor was very robust in complicated biological samples. The authors showed the specificity of this method for free tryptophan using tyrosine, phenylalanine, and two dipeptides as competing chemicals. They established that proteins in biological samples do not interfere with the assay in contrast to other separation-free colorimetric methods, but this indole-hydroxylamine reaction-based tryptophan detection exhibited a poor LOD of $100 \mu \mathrm{M}$ and linear range of up to $600 \mu \mathrm{M}$.

To improve the sensitivity of optical detection of tryptophan, a water-stable metal-organic framework (MOF), ZJU-108, was synthesized as a luminescent sensor. ${ }^{152}$ Owing to a lower singlet level of tryptophan compared with other amino acids, the singlet-singlet Förster energy transfer (S-SFET) mechanism was applied to design a luminescent sensor. By selecting a tryptophan appropriate singlet energy level ligand 6-(4-pyridyl)-terephthalic acid and the biocompatible and inexpensive metal ions, $\mathrm{Zn}^{2+}$ as a node, a luminescent MOF, ZJU-108, was fabricated. As

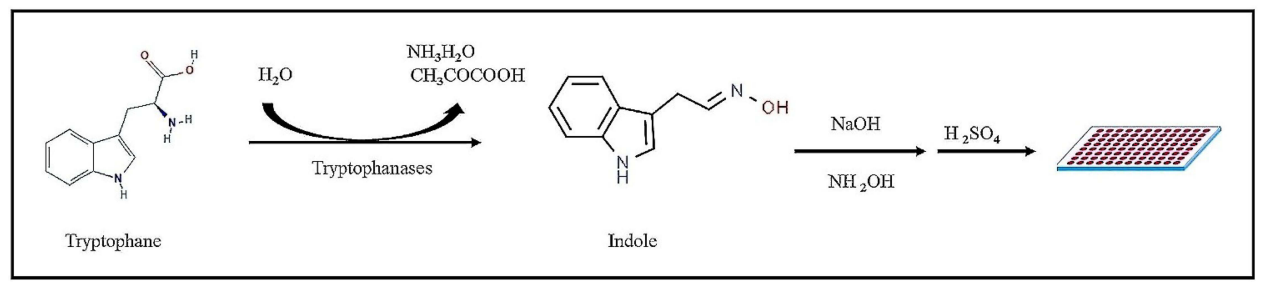

Figure 7 Schematic diagram of high-throughput colorimetric tryptophan assay.

Notes: Tryptophan is converted to indole enzymatically by purified tryptophanases and then used reactivity of indole with hydroxylamine to form a pink color product. Adapted from Talanta, Vol 176, Issue 2018, Wu Y, Wang T, Zhang C, Xing X-H, A rapid and specific colorimetric method for free tryptophan quantification, Pages No. 604609, Copyright (2018), with permission from Elsevier. ${ }^{151}$ 
anticipated, ZJU-108 showed good tryptophan selective luminescence enhancement with a LOD of $42.9 \mathrm{nM}$. Furthermore, ZJU-108 displayed exceptional thermal stability (decomposition temperature of $400^{\circ} \mathrm{C}$ ), water stability (the integrity of its framework after one-month immersion in water), and $\mathrm{pH}$ stability ( $\mathrm{pH}$ range of 1.8 11.7 for $12 \mathrm{~h}$ ). Despite exceptional stability and good LOD, ZJU-108 is an enhancement-based sensor, meaning it is responsive to other amino acid but give a lower signal as compared to tryptophan. In addition, the luminescence with ZJU-108 was evaluated with only individual amino acids and tryptophan, but not with the mixture of various amino acids and tryptophan. Assessment of tryptophan in a mixture of amino acids might lead to high background noise and false results. Therefore, further research is needed to develop a tryptophan-specific sensor.

A selective and sensitive colorimetric method based on surface-enhanced Raman scattering (SERS) and diazo coupling reaction was developed to quantify trace levels of tryptophan in complex biological samples. Surface-enhanced Raman scattering technology provides a sharp spectral peak of small molecules at very low concentrations, ${ }^{152,153}$ which enables to increase the signal intensity by 6-14 times. Tryptophan has low SERS intensity even at the high concentration. Tryptophan combines with naphthalene ethylenediamine (NEDA) molecules to form a bigger composite through a bridge of $-\mathrm{N}^{2+}$ following diazotization, which increases the contact surface of tryptophan and indirectly amplified the SERS signal strength of tryptophan. ${ }^{154}$ The LOD of $20 \mathrm{nM}$, with the linear detection range of $50 \mathrm{nM}$ to $1 \mathrm{mM}$, was achieved by this method. The developed sensor was used to analyze the difference in serum tryptophan concentrations between healthy people and colorectal cancer patients. The results showed a high level of correlation when compared with the data obtained by HPLC. The major chemicals used in the diazo-coupling reaction include sodium nitrite and ammonium sulfamate, which are highly toxic to the environment and humans both. ${ }^{155}$ Especially, sodium nitrite is an extremely powerful oxidizing agent, it may cause hypotension, dysrhythmias, alters consciousness, and limits oxygen transport and delivery in the body through the formation of methemoglobin. ${ }^{156}$ The use of such extreme toxic chemicals should be strongly discouraged and biocompatible chemicals must be used to ensure sustainable development.

Recently, fluorescent chiral sensors with straightforward signals such as enantioselective fluorescence enhancement attracted interest in the determination of enantiomers of amino acids. Wu et al $^{157}$ synthesized polymeric chiral ionic liquid-4 (PCIL-4) through free radical polymerization for enantioselective detection of tryptophan. Primarily, polyethylene (PSVP-3) is chosen as the backbone by using 2azoisobutyronitrile (AIBN) as initiator and cetyltrimethyl ammonium bromide (CTAB). CTAB forms micelle to control homogeneity in ethanol and control the open-ended growth of polymers. Then, phenyl and pyridine groups are physically separated, but meanwhile, allow through-space delocalization effect to fabricate fluorescence polymers. Secondly, phenylalanine derivative, poly(ionic liquid) (S)PCIL-4 (a chiral molecule) with alkyl chloride is linked to the pyridyl nitrogen group to form pyridinium cation. The constructed sensor can afford photoluminescence in the presence of spatial $\pi-\pi$ and ion $-\pi$ interaction. Then, (S)-PCIL-4 can be served as a fluorescent turn-off/on the sensor for chiral recognition of tryptophan in the presence of $\mathrm{Cu}^{2+}$. The linear detection range for tryptophan was $0-200 \mathrm{mM}$ and $0-175$ $\mathrm{mM}$, with an enantiomeric fluorescence difference ratio of 1.08. Nonetheless, this research opens up a possible pathway to synthesize different chiral fluorescent sensors. Subsequently, Pundi et al ${ }^{158}$ synthesized a chiral carbazole sensor (CCS), bearing a "ureacarboxylic acid" binding site, for quantitative fluorescence detection of tryptophan. CCS sensor can act as a reversible fluorescence quenching sensor for $\mathrm{Fe}^{3+}$. Then, the quenched fluorescence of the CCS-Fe ${ }^{3+}$ complex can be recovered upon the addition of tryptophan, leading to quantitative detection of tryptophan in an aqueous solution. CCS showed a low LOD of $0.31 \mu \mathrm{M}$ for tryptophan. Moreover, the tryptophan contents in three commercial pharmaceutical sleep-improving capsules were analyzed by CCS with high accuracy. Further experiments are needed to approve this sensor for evaluation of tryptophan in complex biological matrix-like serum, urine, and food sample.

Colorimetric/fluorescent detection of biomolecules requires spectrophotometric measurement, which are moderately expensive. To reduce the cost of detection, scanometry was used to assess the color change. Scanometry uses a flatbed scanner to measure the reflection of light from the solution. Another advantage of scanometry over spectrophotometry is that the sharpness of the maximum wavelength of the analyte is not a serious problem because the measured color intensity is analyzed based on different color values (red, green and blue, etc.), thus provides a good result even if the samples are turbid. Using scanometry, Jafari et al ${ }^{159}$ developed a fast and inexpensive colorimetric sensor for chiral recognition of tryptophan enantiomers by chitosancapped silver nanoparticles (CS-AgNPs). No color change was observed in CS-AgNPs solution in the presence of D- 
tryptophan, whereas color changed from yellowish to brown in the presence of L-tryptophan. The image of the colored solution was taken using the scanometer and the corresponding color values were obtained using Photoshop software, which subsequently was used for the optimization of the experimental parameters as the analytical signal. Two types of color value systems were investigated: RGB (red, green, and blue values) and CMYK (cyan, magenta, yellow and black values). The color values indicated that L-tryptophan had better interaction than D-tryptophan with chitosancapped silver nanoparticles. The authors postulated that selective formation of hydrogen bonds between CS and tryptophan isomers due to twofold helical conformation of the CS molecule has a possible role in chiral recognition of enantiomers. A good linear relationship between the concentration of L-tryptophan and the effective intensity of the color product was obtained in the concentration range of 13-460 $\mu \mathrm{M}$, and LOD was $2.1 \mu \mathrm{M}$. Fabrication of custom-designed 3D-printed accessories compatible with smartphones and suitable software applications can be useful in transforming scanometry-based tryptophan colorimetric assay to a more sensitive, portable, inexpensive, and easy-to-use smartphone-based system.

\section{Optical Detection of Melatonin}

In this section, we discuss colorimetric/fluorometric assays that are not antibody-dependent, except the chip-based immunochromatographic assays. Array-based sensors utilizing nanomaterials have emerged as impressive tools for detecting analytes such as proteins, antioxidants, explosives, metal ions, and toxic gases. ${ }^{160-164}$ The nanomaterial-based array sensors use cross-reactive sensing elements to produce unique responses in the form of colorful or fluorescent patterns in the presence of different analytes; statistical analyses of the patterns facilitate the qualitative and quantitative analysis of various molecules.

In two separate studies by Huang et al, ${ }^{165}$ manganese oxide $\left(\mathrm{MnO}_{2}\right)$-3,3',5,5'-tetramethylbenzidine (TMB) nanosheets $\left(\mathrm{MnO}_{2}-\mathrm{TMB}\right)$, and sodium hypochlorite $(\mathrm{NaClO})-\mathrm{TMB}$ nanosheets $(\mathrm{NaClO}-\mathrm{TMB})^{166}$ were synthesized to develop multicolor sensor arrays for the analysis of various antioxidants including L-glutathione, melatonin, L-cysteine, UA and AA. ${ }^{165,166}$ The varying reducing powers of the listed antioxidants elicited distinct colorimetric response patterns, which could be observed at wavelengths of 370, 450, and $650 \mathrm{~nm}$. Principal component analyses confirmed that the two colorimetric assays could effectively discriminate between the antioxidants, both in the buffer and in antioxidant-spiked fetal bovine serum (FBS). The LODs for the antioxidants suspended in FBS were $20 \mu \mathrm{M}$ and $1 \mathrm{nM}$ for the $\mathrm{MnO}_{2}-\mathrm{TMB}$ and $\mathrm{NaClO}-\mathrm{TMB}$ sensors, respectively. Notably, these economical $\mathrm{MnO}_{2}$-TMB and $\mathrm{NaClO}-\mathrm{TMB}$ sensors can discriminate between various antioxidants present in FBS, and the results are visible to the naked eye; however, this technique is not functional at clinically relevant concentrations. Significant improvements to the sensitivity of these sensors will be required if they are to be successfully applied for the monitoring and detection of disease, which is the primary purpose of most visual biomolecule sensors.

Another colorimetric/fluorometric sensor was developed for the detection of melatonin in saliva, using 2.3naphthalenedialdehyde $(2,3-\mathrm{Nda})$ as a chromogenic reagent. ${ }^{167}$ Under acidic conditions, 2.3-Nda reacted with melatonin in the presence of iron (III) chloride catalyst; the color of the solution changed from colorless to yellow in a concentration-dependent manner. Furthermore, applying different concentrations of the reactants generated fluorescence due to the large conjugated system of the reaction product. For the colorimetric method, the detection range of melatonin was $2.5-37.5 \mu \mathrm{M}$ with a LOD of $1.288 \mu \mathrm{M}$; the range of melatonin detection for the fluorometric method was $0.01-0.1 \mu \mathrm{M}$ with a LOD of $0.004 \mu \mathrm{M}$. This assay offered higher sensitivity in comparison to other non-enzymatic melatonin detection assays. A potential limitation of this assay was the use of hydrochloric acid to create the acidic conditions for the reaction; hydrochloric acid is highly corrosive and can be harmful to both the environment and the user. ${ }^{168}$ Another drawback is the presence of reactive aldehyde groups on 2.3-Nda, which may easily be attacked by nucleophiles present in the biological samples, such as the sulfhydryl/thiol side-chains of cysteine residues and thiolate. ${ }^{169,170}$ To ensure the reliability of this assay, further investigation into the effects of different concentrations of biological nucleophilic agents would be required.

Recently, an immunochromatographic assay (ICA) was developed for rapid quantitative melatonin detection using AuNPs conjugated to mouse monoclonal antibodies. ${ }^{171}$ The ICA yielded a LOD of $0.185 \mu \mathrm{M}$ as determined by eye, and a LOD of $215.25 \mathrm{nM}$ as determined using a strip reader, for pharmaceutical melatonin samples measured under optimized experimental conditions. The linear detection range was from $215.25 \mathrm{nM}$ to $42 \mu \mathrm{M}$, which was comparable to standard liquid chromatography. This 
ICA could be an excellent tool for determining melatonin levels in foods; however, the development of a lateral flow ICA device for melatonin detection with higher sensitivity is essential. The main culprit for low sensitivity in ICAs is a transient reaction triggered by capillary action on the nitrocellulose membrane (NCM). This transient reaction causes low binding rates between probe-labeled targets and the immobilized antibodies on the nitrocellulose membrane. Modifying the immobilized capture antibodies may increase their binding efficiency, which is currently limited by the protein-binding capacity of the nitrocellulose membrane. Thus, the development of novel materials with higher binding affinities may overcome the limited protein-binding capacity of the nitrocellulose membranes and further improve the LOD and range of detection of ICAs.

\section{Challenges and Future Perspectives}

Due to the widespread availability of tryptophan in several proteins, there is still a need to develop highly specific sensors, which are not susceptible to the matrix and $\mathrm{pH}$. On the other hand, the biggest challenge for melatonin researchers is to evaluate circulatory concentrations of $<8.6 \mathrm{pM}$ in the presence of metabolites with similar molecular structures in highly diverse and complex matrices. Despite recent efforts, significant improvements to the LOD of current assays will be required to deliver inexpensive, simple, point-of-care (POC) testing. The emerging sensors discussed in this review offer many advantages over conventional tryptophan and melatonin testing methods such as simplicity, co-detection, and portability; however, reproducibility, stability, the detection range, and LOD are still matters of concern. To improve assay reliability, researchers should report inter- and intra-assay variability in assay sensitivity.

The number of recent advancements in electrochemical sensors reflects the significance of this field, and the power, flexibility, and convenience that electrochemistry can offer to the detection of biomolecules. However, each of the electrochemical sensors was subject to the common issue of electrode surface biofouling, especially during the analysis of complex biological samples. To evaluate biomolecules electrochemically, the electrode surfaces must physically interact with working solutions, which include clinical samples such as plasma, serum, sweat, saliva, or urine. The interaction between the electrodes and the solution will, immediately or eventually, lead to the adsorption and adhesion of biomolecules onto the electrode surface; this inevitable biofouling interferes with the sensitivity and selectivity of the sensors. ${ }^{172,173}$ Therefore, a key requirement for developing a robust and sensitive electrochemical sensor is the synthesis or discovery of powerful anti-fouling material. Novel antifouling materials could be amalgamated with the present sensing/electroactive materials to fabricate a stable sensor. In an attempt to develop a foul-proof bioelectrode, the surface of an electrode was co-deposited with polydopamine and hyaluronic acid. The deposition of polydopamine facilitated the absorption of hyaluronic acid, which exhibits antifouling properties, onto the electrode surface. ${ }^{174}$ Alternatively, a novel material with both electroactive and anti-fouling activities may also be synthesized. For example, newly discovered conjugated polymers such as poly(sulfobetaine-3,4-ethylenedioxythiophene) exhibit excellent conductivity and antifouling/antimicrobial properties. ${ }^{175}$ Another prominent issue facing electrochemical sensor production is a lack of study regarding their shelf life. Typically, electrodes destined for electrochemical detection are developed using novel nanocomposites, yet the nanocomposite shelf-lives are rarely reported. Comprehensive analyses examining the shelf lives of nanocomposites and electrodes decorated with nanocomposites in various solutions should be conducted. Furthermore, the development of nanocomposites requires skillful handling and expensive materials such as $\mathrm{Pd} ;{ }^{88}$ therefore, simpler protocols with cheaper materials should be developed to reduce the overall price and complexity of analyte detection. Before using nanocomposite-based electrochemical sensors, studying their biocompatibility and cytotoxicity is also mandatory to ensure their in vivo applicability.

Although robust techniques are available for tryptophan and melatonin detection, the majority are laboratory-based, expensive, and require technical training. The clinical significance of tryptophan and melatonin has until recently been underestimated; however, recent research has increasingly demonstrated their key functions in human health and disease. Mental health and sleep disorders, jet lag, and shift work present novel problems associated with modern life; detection of their associated ailments usually take place only once they have entered their chronic phase.

Moreover, artificial light is a potent circadian rhythm disruptor that reduces melatonin levels with serious consequences. $^{177-180}$ POC measurements of tryptophan and melatonin levels could enable the early diagnosis of circadian disruption-related physiological and psychological disorders. In addition, sensing both melatonin and tryptophan in real-time might provide key insights into 
their role in immunomodulation, inflammatory disease, and inflammatory responses to pandemic diseases such as COVID-19. ${ }^{180-183}$ Thus, nanoelectrodes for real-time detection that are easily synthesized, durable, inexpensive, eco-friendly, biocompatible, selective, and sensitive are urgently needed. Recently, microelectromechanical neurotools for non-invasive ultrasound brain stimulation were developed to treat brain disorders. ${ }^{184}$ Similarly, with the help of big data analysis and artificial intelligence, wearable micro/nanoelectromechanical sensors could be developed to study the sleep-wake cycle and accurately quantify circadian disruption. ${ }^{185}$ Strategies to diagnose circadian disruptions accurately using wearable sensors could potentially lead to novel therapeutic interventions. ${ }^{185,186}$ Another potentially fruitful avenue of tryptophan and melatonin detection research is the development of lateral flow ICAs. In recent years, lateral flow ICAs have attained considerable commercial success, which has a positive impact on disease diagnosis. However, there is a scarcity of lateral flow ICAs available for melatonin detection due to the need for very high sensitivity. Lateral flow ICAs are typically performed by examining color changes by the naked eye. Optical (colorimetric, fluorescence, chemiluminescence, and surface-enhanced Raman scattering), thermal (thermal imaging, photoacoustics, and speckle imaging), magnetic (magnetic nanoparticle quantification, giant magnetoresistance, and tunneling magnetoresistance), and electrochemical (various voltammetry and electro-chemiluminescence) technologies could be utilized to develop high-sensitivity lateral flow ICAs to quantify melatonin in biological samples for POC testing. ${ }^{187}$

\section{Conclusion}

In the present review, we have objectively summarized both electrochemical and optical sensors for tryptophan and melatonin detection, providing a scientific opinion on the future of the field. Given the vast number of publications in this field, we have focused on high-sensitivity methods developed within the last 6 years. Even with this focus, we will have inevitably and unintentionally excluded some emerging technologies. Over the past few decades, there has been significant interest in the development of tryptophan and melatonin detection techniques using immunoassays, spectrometry, HPLC, MS, colorimetry, fluorometry, and electroanalytical sensors. Among these, electroanalytical detection tools present several advantages, including low LODs and high range, low interference, cost-effectiveness, fast response times, and suitability for quantitative and analytical applications. Future work on electrochemical sensors may involve the optimization of LOD and sensitivity, the development of an integrated detection system, and the monitoring of biomolecules in vivo. Furthermore, to compensate for the lack of output signal from the MIT, the fluorescent or highly electroactive materials should be coupled with the high selectivity of MIT to develop ultrasensitive and selective sensors used for the detection of tryptophan and melatonin. In summary, further work is needed to achieve mass production with MIT, and research into the mechanism and the characterization of "on-off" fluorophore sensors are still highly limited and require further development. Despite notable recent advancements in electrochemical sensor development, attempts to design and explore novel techniques for eco-friendly, cheaper, durable, and faster sensing tools should be actively pursued. For colorimetric/fluorometric sensors and lateral flow devices to achieve commercial success, significant improvements to sensitivity are needed. We also want to highlight that except modifications of immunoassays, there is a substantial lack of research regarding optical detection of tryptophan and melatonin. There is an immediate need to develop novel colorimetric and fluorescent materials for optical sensing of tryptophan and melatonin. Finally, commercialization efforts and application-driven collaborations of different disciplines will be critical for future developments.

\section{Abbreviations}

AA, ascorbic acid; cELISA, competitive enzyme-linked immunosorbent assay; CV, cyclic voltammetry; Cys, cysteine; DPV, differential pulse voltammetry; DSI-MS, droplet spray ionization mass spectrometry; EIS, electrochemical impedance spectroscopy; FSCV, fast-scan cyclic voltammetry; FBS, fetal bovine serum; GC-MS, gas chromatography-mass spectrometry; GSH, glutathione; HPLC, high-performance liquid chromatography; ICA, immunochromatographic assay; LOD, limit of detection; LC-MS/ MS, liquid chromatography with tandem mass spectrometry; PS, pharmaceutical samples; RIA, radioimmunoassay; SWV, square wave voltammetry; TMB, 3.3',5,5'-tetramethylbenzidine; UHPLC/HRMS, ultra-high-performance liquid chromatography coupled to high-resolution mass spectrometry; UA, uric acid.

\section{Acknowledgment}

The authors would like to acknowledge the invaluable support and critical comments of members in the 
'Biological Clock \& Aging Control' laboratory at Inje University, Korea.

\section{Author Contributions}

All authors made a significant contribution to the work reported, whether that is in the conception, study design, execution, acquisition of data, analysis and interpretation, or in all these areas; took part in drafting, revising or critically reviewing the article; gave final approval of the version to be published; have agreed on the journal to which the article has been submitted; and agree to be accountable for all aspects of the work.

\section{Funding}

There is no funding to report.

\section{Disclosure}

The authors have no potential conflicts of interest to disclose.

\section{References}

1. Ciuris C, Lynch HM, Wharton C, Johnston CS. A comparison of dietary protein digestibility, based on DIAAS scoring, in vegetarian and non-vegetarian athletes. Nutrients. 2019;11(12):3016. doi: $10.3390 /$ nu11123016

2. Delgado-Andrade C, Rufián-Henares JA, Jiménez-Pérez S, Morales FJ. Tryptophan determination in milk-based ingredients and dried sport supplements by liquid chromatography with fluorescence detection. Food Chem. 2006;98(3):580-585. doi:10.1016/ j.foodchem.2005.07.036

3. Badawy A-B. Plasma free tryptophan revisited: what you need to know and do before measuring it. J Psychopharmacol. 2010;24 (6):809-815. doi:10.1177/0269881108098965

4. Richard DM, Dawes MA, Mathias CW, Acheson A, HillKapturczak N, Dougherty DM. L-tryptophan: basic metabolic functions, behavioral research and therapeutic indications. Int $J$ Tryptophan Res. 2009;2(IJTR):S2129. doi:10.4137/IJTR.S2129

5. Singh R. Role of tryptophan in health and disease: systematic review of the anti-oxidant, anti-inflammation, and nutritional aspects of tryptophan and its metabolites. World Heart $J$. 2019;11(2):161-178.

6. Gao K, Mu C. Tryptophan metabolism: a link between the gut microbiota and brain. Adv Nutrition. 2019;11(3):709-723. doi:10.1093/advances/nmz127

7. Bech-Andersen S. Determination of tryptophan with HPLC after alkaline hydrolysis in autoclave using $\alpha$-methyl-tryptophan as internal standard. Acta Agriculturae Scandinavica. 1991;41 (3):305-309. doi:10.1080/00015129109439913

8. la Cour R, Jørgensen H, Schjoerring JK. Improvement of Tryptophan analysis by liquid chromatography-single quadrupole mass spectrometry through the evaluation of multiple parameters. Front Chem. 2019;19(7):797-822. doi:10.3389/ fchem.2019.00797

9. Zawilska JB, Nowak JZ. Melatonin: from biochemistry to therapeutic applications. Pol J Pharmacol. 1999;51(1):3-23.

10. Chattoraj A, Liu T, Zhang LS, Huang Z, Borjigin J. Melatonin formation in mammals: in vivo perspectives. Rev Endocr Metab Disord. 2009;10(4):237-243. doi:10.1007/s11154-009-9125-5
11. Utiger RD. Melatonin - the Hormone of Darkness. $N$ Engl J Med. 1992;327(19):1377-1379. doi:10.1056/NEJM199211053271909

12. Sánchez-Vázquez FJ, López-Olmeda JF, Vera LM, Migaud H, López-Patiño Ma, Míguez JM. Environmental Cycles, melatonin, and circadian control of stress response in fish. Front Endocrinol (Lausanne). 2019;11(10):279-293. doi:10.3389/fendo.2019.00279

13. Zawilska JB. Melatonin as a chemical indicator of environmental light-dark cycle. Acta Neurobiol Exp. 1996;56(3):757-767.

14. Falcón J, Besseau L, Fuentès M, Sauzet S, Magnanou E, Boeuf G. Structural and functional evolution of the pineal melatonin system in vertebrates. Ann N Y Acad Sci. 2009;1163(1):101-111. doi:10.1111/j.1749-6632.2009.04435.x

15. Zhang R, Wang $\mathrm{X}$, Ni L, et al. COVID-19: melatonin as a potential adjuvant treatment. Life Sci. 2020;250(1):117583. doi:10.1016/j.lfs.2020.117583

16. Shneider A, Kudriavtsev A, Vakhrusheva A. Can melatonin reduce the severity of COVID-19 pandemic? Int Rev Immunol. 2020;39(4):153-162. doi:10.1080/08830185.2020.1756284

17. Reiter RJ, Abreu-Gonzalez P, Marik PE, Dominguez-Rodriguez A. Therapeutic algorithm for use of melatonin in patients with COVID19. Front Med. 2020;7(1):226. doi:10.3389/fmed.2020.00226

18. Kennaway DJ, Voultsios A. Circadian Rhythm of free melatonin in human plasma. J Clin Endocrinol Metab. 1998;83(3):1013-1015.

19. Hsing AW, Meyer TE, Niwa S, Quraishi SM, Chu LW. Measuring serum melatonin in epidemiologic studies. Cancer Epidemiol Biomarkers Prevention. 2010;19(4):932-937. doi:10.1158/10559965.EPI-10-0004

20. Kim Y, Lee S-J, Park C-S, et al. The mediating effect of eveningness on the indirect relationships between shorter sleep duration, inattention, depression with smartphone addiction tendency. Chronobiol Med. 2020;2(1):32-40. doi:10.33069/cim.2020.0004

21. Kripke DF. Delayed Circadian rhythms and pars tuberalis dysfunction in mood disorders. Chronobiol Med. 2020;2(1):1-2. doi:10.33069/cim.2020.0001

22. Sundberg I, Rasmusson AJ, Ramklint M, Just D, Ekselius L, Cunningham JL. Daytime melatonin levels in saliva are associated with inflammatory markers and anxiety disorders. Psychoneuroendocrinology. 2020;112(1):104514. doi:10.1016/j. psyneuen.2019.104514

23. Gomez F, Gatica Hernández I, Silva M, Cerutti S. Analytical Trends for the determination of melatonin and precursors in plants. J Pineal Res. 2016;1(1):53-68.

24. De Almeida EA, Di Mascio P, Harumi T, et al. Measurement of melatonin in body fluids: standards, protocols and procedures. Childs Nerv Syst. 2011;27(6):879-891. doi:10.1007/s00381-0101278-8

25. Gooneratne NS, Edwards AYZ, Zhou C, Cuellar N, Grandner MA, Barrett JS. Melatonin pharmacokinetics following two different oral surge-sustained release doses in older adults. $J$ Pineal Res. 2012;52(4):437-445. doi:10.1111/j.1600079X.2011.00958.x

26. EFSA Panel on Dietetic Products N, Allergies. Scientific Opinion on the substantiation of a health claim related to melatonin and reduction of sleep onset latency (ID 1698, 1780, 4080) pursuant to Article 13 (1) of Regulation (EC) No 1924/2006. EFSA J. 2011;9(6):2241. doi:10.2903/j.efsa.2011.2241

27. Muñoz JLP, Ceinos RM, Soengas JL, Míguez JM. A simple and sensitive method for determination of melatonin in plasma, bile and intestinal tissues by high performance liquid chromatography with fluorescence detection. $J$ Chromatogr B. 2009;877 (22):2173-2177. doi:10.1016/j.jchromb.2009.06.001

28. Zhang L, Li Y, Zhou H, Li L, Wang Y, Zhang Y. Determination of eight amino acids in mice embryonic stem cells by pre-column derivatization HPLC with fluorescence detection. $J$ Pharm Biomed Anal. 2012;66(1):356-358. doi:10.1016/j. jpba.2012.03.014 
29. Dario MF, Freire TB, de Oliveira Pinto CAS, Prado MSA, Baby AR, Velasco MVR. Tryptophan and kynurenine determination in human hair by liquid chromatography. $J$ Chromatogr $B$. 2017;1065(1):59-62. doi:10.1016/j.jchromb.2017.09.020

30. Fourtillan J, Gobin P, Faye B, Girault J. A highly sensitive assay of melatonin at the femtogram level in human plasma by gas chromatography/negative ion chemical ionization mass spectrometry. Biol Mass Spectrom. 1994;23(8):499-509. doi:10.1002/ bms. 1200230807

31. Markey SP, Boni RL, Yergey JA, Heyes MP. Mass spectrometric determinations of tryptophan and its metabolites. In: Schwarcz R, Young SN, Brown RR. Kynurenine and Serotonin Pathways: Progress in Tryptophan Research. Springer New York; 1991:41-50.

32. Kim YO, Chung HJ, Chung ST, et al. Determination of melatonin in biological samples by capillary electrophoresis. J Chromatogr A. 1999;850(1-2):369-374. doi:10.1016/S0021-9673(99)00167-3

33. Zhao M, Zhou M-F, Feng H, Cong -X-X, Wang X-L. Determination of tryptophan, glutathione, and uric acid in human whole blood extract by capillary electrophoresis with a one-step electrochemically reduced graphene oxide modified microelectrode. Chromatographia. 2016;79(13):911-918. doi:10.1007/s10337-016-3115-z

34. Lewy A, Markey S. Analysis of melatonin in human plasma by gas chromatography negative chemical ionization mass spectrometry. Science. 1978;201(4357):741-743. doi:10.1126/ science. 675255

35. Kałuzna-Czaplinska J, Michalska M, Rynkowski J. Determination of tryptophan in urine of autistic and healthy children by gas chromatography/mass spectrometry. Med Sci Monitor. 2010;16 (10):CR488-CR492.

36. Kennaway DJ. A critical review of melatonin assays: past and present. J Pineal Res. 2019;67(1):e12572. doi:10.1111/jpi.12572

37. Liu G, Huang X, Li L, et al. Recent advances and perspectives of molecularly imprinted polymer-based fluorescent sensors in food and environment analysis. Nanomaterials. 2019;9(7):1030. doi:10.3390/nano9071030

38. Mosbach K. Molecular imprinting. Trends Biochem Sci. 1994;19 (1):9-14. doi:10.1016/0968-0004(94)90166-X

39. Komiyama M, Takeuchi $T$, Mukawa $T$, Asanuma $H$. Nanoarchitectonics to prepare practically useful artificial enzymes. Mol Imprinting. 2003;1;148.

40. Gupta A, Kumar N. A review of mechanisms for fluorescent "turn-on" probes to detect $\mathrm{Al}^{3+}$ ions. RSC $A d v$. 2016;6 (108):106413-106434. doi:10.1039/C6RA23682K

41. Huang Y, Xing J, Gong Q, et al. Reducing aggregation caused quenching effect through co-assembly of PAH chromophores and molecular barriers. Nat Commun. 2019;10(1):169. doi:10.1038/ s41467-018-08092-y

42. Scozzari A. Electrochemical sensing methods: a brief review. Springer Netherlands. 2008;1(1):335-351.

43. Elgrishi N, Rountree KJ, McCarthy BD. A practical beginner's guide to cyclic voltammetry. $J$ Chem Educ. 2018;95(2):197-206. doi:10.1021/acs.jchemed.7b00361

44. Ramli NI, Ismail NAB, Abd-Wahab F, Salim WWAW. Cyclic voltammetry electrical impedance spectroscopy of electrodes modified with PEDOT: PSS-reduced graphene oxide composite. Transparent Conducting Films IntechOpen. 2018;1(1):1.

45. Tiwari MP, Prasad A. Molecularly imprinted polymer based enantioselective sensing devices: a review. Anal Chim Acta. 2015;853(1):1-18. doi:10.1016/j.aca.2014.06.011

46. Gholivand MB, Jalalvand AR, Goicoechea HC, Gargallo R, Skov T. Chemometrics: an important tool for monitoring interactions of vitamin B7 with bovine serum albumin with the aim of developing an efficient biosensing system for the analysis of protein. Talanta. 2015;132(1):354-365. doi:10.1016/j.talanta.2014.09.019
47. Chooto P. Cyclic voltammetry and its applications. Voltammetry. IntechOpen. 2019;p.1.

48. Zhang Y, Waterhouse GIN, Xiang Z. A highly sensitive electrochemical sensor containing nitrogen-doped ordered mesoporous carbon (NOMC) for voltammetric determination of 1-tryptophan. Food Chem. 2020;326:126976. doi:10.1016/j. foodchem.2020.126976

49. Apetrei IM, Apetrei C. Voltammetric determination of melatonin using a graphene-based sensor in pharmaceutical products. Int $J$ Nanomedicine. 2016;11:1859-1866. doi:10.2147/IJN.S104941

50. Martín A, Escarpa A. Graphene: the cutting-edge interaction between chemistry and electrochemistry. TrAC Trends Analytical Chem. 2014;56:13-26. doi:10.1016/j.trac.2013.12.008

51. Stankovich S, Dikin DA, Dommett GHB, et al. Graphene-based composite materials. Nature. 2006;442(7100):282-286. doi:10.1038/nature04969

52. Hensley AL, Colley AR, Ross AE. Real-Time Detection of Melatonin Using Fast-Scan Cyclic Voltammetry. Anal Chem. 2018;90(14):8642-8650. doi:10.1021/acs.analchem.8b01976

53. Radi A, Bekhiet G. Voltammetry of melatonin at carbon electrodes and determination in capsules. Bioelectrochem Bioenerg. 1998;45 (2):275-279. doi:10.1016/S0302-4598(97)00101-3

54. Levent A. Electrochemical determination of melatonin hormone using a boron-doped diamond electrode. Diam Relat Mater. 2012;21:114-119. doi:10.1016/j.diamond.2011.10.018

55. Xiao-Ping W, Lan Z, Wen-Rong L, Jian-Ping D, Hong-Qing C, Guo-Nan C. Study on the electrochemical behavior of melatonin with an activated electrode. Electroanalysis. 2002;14(23):16541660. doi:10.1002/elan.200290007

56. Topçu E, Dağcı Kıranşan K. Electrochemical simultaneous sensing of melatonin and ascorbic acid at a novel flexible B-RGO composite paper electrode. Diam Relat Mater. 2020;105:107811. doi:10.1016/j.diamond.2020.107811

57. Ozkan SA, Kauffmann J-M ZP. Electroanalysis in biomedical and pharmaceutical sciences: voltammetry, amperometry, biosensors, applications. Springer; 2015.

58. Tadayon F, Sepehri Z. A new electrochemical sensor based on a nitrogen-doped graphene $/ \mathrm{CuCo}_{2} \mathrm{O}_{4}$ nanocomposite for simultaneous determination of dopamine, melatonin and tryptophan. RSC Adv. 2015;5(80):65560-65568. doi:10.1039/C5RA12020A

59. Ou L, Song B, Liang H, et al. Toxicity of graphene-family nanoparticles: a general review of the origins and mechanisms. Part Fibre Toxicol. 2016;13(1):57.

60. Guo X, Mei N. Assessment of the toxic potential of graphene family nanomaterials. J Food Drug Analysis. 2014;22(1):105115. doi:10.1016/j.jfda.2014.01.009

61. Fadeel B, Bussy C, Merino S, et al. Safety assessment of graphene-based materials: focus on human health and the environment. ACS Nano. 2018;12(11):10582-10620. doi:10.1021/ acsnano. $8 \mathrm{~b} 04758$

62. Zeinali H, Bagheri H, Monsef-Khoshhesab Z, Khoshsafar H, Hajian A. Nanomolar simultaneous determination of tryptophan and melatonin by a new ionic liquid carbon paste electrode modified with $\mathrm{SnO}_{2}-\mathrm{Co}_{3} \mathrm{O}_{4} @ \mathrm{rGO}$ nanocomposite. Materials Sci Eng. 2017;71:386-394. doi:10.1016/j.msec.2016.10.020

63. Khan ZA, Yumnamcha T, Rajiv C, et al. Melatonin biosynthesizing enzyme genes and clock genes in ovary and whole brain of zebrafish (Danio rerio): differential expression and a possible interplay. Gen Comp Endocrinol. 2016;233:16-31. doi:10.1016/ j.ygcen.2016.05.014

64. Rajiv C, Sanjita Devi H, Mondal G, et al. Cloning, phylogenetic analysis and tissue distribution of melatonin bio-synthesizing enzyme genes (Tph1, Aanat1, Aanat2 and Hiomt) in a tropical carp, Catla catla. Biol Rhythm Res. 2017;48(3):371-386. doi:10.1080/09291016.2016.1263019 
65. Sanjita Devi H, Rajiv C, Mondal G, et al. Melatonin bio-synthesizing enzyme genes (Tph1, Aanat1, Aanat2, and Hiomt) and their temporal pattern of expression in brain and gut of a tropical carp in natural environmental conditions. Cogent Biol. 2016;2 (1):1230337. doi:10.1080/23312025.2016.1230337

66. MacDonald S, Roscoe S. Electrochemical oxidation reactions of tyrosine, tryptophan and related dipeptides. Electrochim Acta. 1997;42(8):1189-1200. doi:10.1016/S0013-4686(96)00285-X

67. Mukdasai S, Poosittisak S, Ngeontae W, Srijaranai S. A highly sensitive electrochemical determination of 1-tryptophan in the presence of ascorbic acid and uric acid using in situ addition of tetrabutylammonium bromide on the B-cyclodextrin incorporated multi-walled carbon nanotubes modified electrode. Sens Actuators B Chem. 2018;272:518-525. doi:10.1016/j.snb.2018.06.014

68. Boruń A, Bald A. Conductometric studies of sodium tetraphenylborate, tetrabutylammonium bromide, and sodium tetrafluoroborate in $\mathrm{N}, \mathrm{N}$-dimethylformamide at temperatures from (283.15 to 318.15$) \mathrm{K}$. J Chem Eng Data. 2012;57(7):2037-2043. doi:10.1021/je300252d

69. Imai Y, Shimamoto K, Takiue T, Matsubara H, Aratono M. Study on surface adsorption from cationic surfactant-electrolyte mixed aqueous solution including $\mathrm{BF}_{4}^{-}$ion. Colloid Polym Sci. 2010;288(9):1005-1011. doi:10.1007/s00396-010-2221-y

70. Pokrovskaya MV. Graphene 'outperforms' carbon nanotubes as reinforcement in composite materials. Additives Polymers. 2010;2010(6):10-11. doi:10.1016/S0306-3747(10)70107-X

71. Li J, Jiang J, Xu Z, et al. Facile synthesis of $\mathrm{Pd}-\mathrm{Cu} @ \mathrm{Cu}_{2} \mathrm{O} / \mathrm{N}-\mathrm{RGO}$ hybrid and its application for electrochemical detection of tryptophan. Electrochim Acta. 2018;260:526-535. doi:10.1016/j. electacta.2017.12.125

72. Haldorai Y, Yeon S-H, Huh YS, Han Y-K. Electrochemical determination of tryptophan using a glassy carbon electrode modified with flower-like structured nanocomposite consisting of reduced graphene oxide and $\mathrm{SnO}_{2}$. Sens Actuators $B$ Chem. 2017;239:1221-1230. doi:10.1016/j.snb.2016.09.119

73. O'Donoghue M. A Guide to Man-made Gemstones. Van Nostrand Reinhold. 1983;28-32.

74. Grishin DV, Zhdanov DD, Pokrovskaya MV, Sokolov NN. Damino acids in nature, agriculture and biomedicine. All Life. 2020;13(1):11-22. doi:10.1080/21553769.2019.1622596

75. Palego L, Betti L, Rossi A, Giannaccini G. Tryptophan biochemistry: structural, nutritional, metabolic, and medical aspects in humans. J Amino Acids. 2016;2016:8952520. doi:10.1155/2016/ 8952520

76. Wirthgen E, Leonard AK, Scharf C, Domanska G. The immunomodulator 1-methyltryptophan drives tryptophan catabolism toward the kynurenic acid branch. Front Immunol. 2020;11(1):313. doi:10.3389/ fimmu.2020.00313

77. Kobayashi K, Maezawa T, Tanaka H, et al. The identification of d-tryptophan as a bioactive substance for postembryonic ovarian development in the planarian Dugesia ryukyuensis. Sci Rep. 2017;7(1):45175. doi:10.1038/srep45175

78. Chen F, Niu X, Yang X, et al. Self-assembled reduced graphene oxide/polyaniline/sodium carboxymethyl cellulose nanocomposite for voltammetric recognition of tryptophan enantiomers. $J$ Mater Sci. 2021;32(9):11791-11804.

79. Advani SG, Hsiao K-T. Manufacturing techniques for polymer matrix composites (PMCs). Elsevier. 2012;3(1):1.

80. Guo Q, Ghadiri R, Weigel T, et al. Comparison of in situ and ex situ methods for synthesis of two-photon polymerization polymer nanocomposites. Polymers. 2014;6:2037-2050. doi:10.3390/ polym6072037

81. Gomez FJV, Martín A, Silva MF, Escarpa A. Screen-printed electrodes modified with carbon nanotubes or graphene for simultaneous determination of melatonin and serotonin. Microchimica Acta. 2015;182(11):1925-1931. doi:10.1007/s00604-015-1520-x
82. Selvam SP, Hansa M, Yun K. Simultaneous differential pulse voltammetric detection of uric acid and melatonin based on a self-assembled Au nanoparticle-- $\mathrm{MoS}_{2}$ nanoflake sensing platform. Sens Actuators B Chem. 2020;307:127683. doi:10.1016/j.snb.2020.127683

83. Vaughan G. Melatonin in humans. Pineal Res Rev. 1984;2:141201.

84. Karasek M, Winczyk K. Melatonin in humans. J Physiol Pharmacol. 2006;57(5):19-39.

85. Arendt J. Melatonin and the Mammalian Pineal Gland. Springer Science \& Business Media; 1994:78.

86. Arendt J, Bojkowski C, Franey C, Wright J, Marks V. Immunoassay of 6-hydroxymelatonin sulfate in human plasma and urine: abolition of the urinary 24-hour rhythm with atenolol. J Clin Endocrinol Metab. 1985;60(6):1166-1173. doi:10.1210/ jcem-60-6-1166

87. Nowak R, McMillen I, Redman J, Short R. The correlation between serum and salivary melatonin concentrations and urinary 6-hydroxymelatonin sulphate excretion rates: two non-invasive techniques for monitoring human circadian rhythmicity. Clin Endocrinol (Oxf). 1987;27(4):445-452. doi:10.1111/j.1365-2265.1987.tb01172.x

88. Rajkumar C, Veerakumar P, Chen SM, Thirumalraj B, Liu SB. Facile and novel synthesis of palladium nanoparticles supported on a carbon aerogel for ultrasensitive electrochemical sensing of biomolecules. Nanoscale. 2017;9(19):6486-6496. doi:10.1039/ C7NR00967D

89. Tavakkoli N, Soltani N, Shahdost-Fard F, Ramezani M, Salavati H, Jalali MR. Simultaneous voltammetric sensing of acetaminophen, epinephrine and melatonin using a carbon paste electrode modified with zinc ferrite nanoparticles. Mikrochim Acta. 2018;185(10):479. doi:10.1007/s00604-018-3009-x

90. Soltani N, Tavakkoli N, Shahdost-Fard F, Salavati H, Abdoli F. A carbon paste electrode modified with $\mathrm{Al}_{2} \mathrm{O}_{3}{ }^{-}$supported palladium nanoparticles for simultaneous voltammetric determination of melatonin, dopamine, and acetaminophen. Mikrochim Acta. 2019;186(8):540. doi:10.1007/s00604-019-3541-3

91. Duan D, Ding Y, Li L, Ma G. Rapid quantitative detection of melatonin by electrochemical sensor based on carbon nanofibers embedded with FeCo alloy nanoparticles. J Electroanal Chem. 2020;873:114422. doi:10.1016/j.jelechem.2020.114422

92. Arnao MB, Hernández-Ruiz J. Melatonin: a new plant hormone and/or a plant master regulator? Trends Plant Sci. 2019;24(1):3848. doi:10.1016/j.tplants.2018.10.010

93. $\mathrm{Hu} \mathrm{W}$, Yang $\mathrm{H}$, Tie $\mathrm{W}$, et al. Natural variation in banana varieties highlights the role of melatonin in postharvest ripening and quality. J Agric Food Chem. 2017;65(46):9987-9994. doi:10.1021/ acs.jafc. $7 \mathrm{~b} 03354$

94. Liu Y, Li M, Li H, et al. In Situ Detection of Melatonin and Pyridoxine in Plants Using a CuO-Poly(1-lysine)/Graphene-Based Electrochemical Sensor. ACS Sustain Chem Eng. 2019;7 (24):19537-19545. doi:10.1021/acssuschemeng.9b04609

95. Barker GC, Gardner AW. Forty years of square-wave polarography. Analyst. 1992;117(12):1811-1828. doi:10.1039/an9921701811

96. Ramaley L, Krause MS. Theory of square wave voltammetry. Anal Chem. 1969;41(11):1362-1365. doi:10.1021/ac60280a005

97. Mirceski V, Gulaboski R, Lovric M, Bogeski I, Kappl R, Hoth M. Square-wave voltammetry: a review on the recent progress. Electroanalysis. 2013;25(11):2411-2422. doi:10.1002/elan.201300369

98. Favaro G, Fiorani M. Determination of pharmaceutical thiols by liquid chromatography with electrochemical detection: use of an electrode with a conductive carbon cement matrix, chemically modified with cobalt phthalocyanine. Anal Chim Acta. 1996;332 (2-3):249-255. doi:10.1016/0003-2670(96)00265-6

99. Beitollahi H, Sheikhshoaie I. Electrocatalytic and simultaneous determination of isoproterenol, uric acid and folic acid at molybdenum (VI) complex-carbon nanotube paste electrode. Electrochim Acta. 2011;56 (27):10259-10263. doi:10.1016/j.electacta.2011.09.017 
100. Janda P, Weber J, Dunsch L, Lever A. Detection of ascorbic acid using a carbon fiber microelectrode coated with cobalt tetramethylpyridoporphyrazine. Anal Chem. 1996;68(6):960-965. doi:10.1021/ac950323r

101. Tajik S, Taher MA, Beitollahi H. First report for electrochemical determination of levodopa and cabergoline: application for determination of levodopa and cabergoline in human serum, urine and pharmaceutical formulations. Electroanalysis. 2014;26(4):796806. doi:10.1002/elan.201300589

102. Beitollahi H, Mohammadi S. Selective voltammetric determination of norepinephrine in the presence of acetaminophen and tryptophan on the surface of a modified carbon nanotube paste electrode. Materials Sci Eng. 2013;33(6):3214-3219. doi:10.1016/j.msec.2013.03.050

103. Beitollahi H, Gholami A, Ganjali MR. Preparation, characterization and electrochemical application of $\mathrm{Ag}-\mathrm{ZnO}$ nanoplates for voltammetric determination of glutathione and tryptophan using modified carbon paste electrode. Materials Sci Eng. 2015;57:107112. doi:10.1016/j.msec.2015.07.034

104. Erra Díaz F, Dantas E, Geffner J. Unravelling the interplay between extracellular acidosis and immune cells. Mediators Inflamm. 2018;2018:1218297. doi:10.1155/2018/1218297

105. Rumbus $Z$, Toth E, Poto L, et al. Bidirectional relationship between reduced blood $\mathrm{pH}$ and acute pancreatitis: a translational study of their noxious combination. Front Physiol. 2018;9:1360. doi:10.3389/fphys.2018.01360

106. Zakharov S, Nurieva O, Kotikova K, Belacek J, Navratil T, Pelclova D. Positive serum ethanol concentration on admission to hospital as the factor predictive of treatment outcome in acute methanol poisoning. Monatsh. Chem. 2017;148(3):409-419.

107. Beitollahi H, Ghofrani Ivari S, Alizadeh R, Hosseinzadeh R. Preparation, characterization and electrochemical application of $\mathrm{ZnO}-\mathrm{CuO}$ nanoplates for voltammetric determination of captopril and tryptophan using modified carbon paste electrode. Electroanalysis. 2015;27(7):1742-1749. doi:10.1002/elan.201500016

108. Babaei A, Zendehdel M, Khalilzadeh B, Taheri A. Simultaneous determination of tryptophan, uric acid and ascorbic acid at iron (III) doped zeolite modified carbon paste electrode. Colloids Surf $B \quad$ Biointerfaces. 2008;66(2):226-232. doi:10.1016/j. colsurfb.2008.06.017

109. Liu B, Ouyang X, Ding Y, Luo L, Xu D, Ning Y. Electrochemical preparation of nickel and copper oxides-decorated graphene composite for simultaneous determination of dopamine, acetaminophen and tryptophan. Talanta. 2016;146:114-121. doi:10.1016/j. talanta.2015.08.034

110. Motaghedifard M, Behpour M, Amani A. Electrochemical growth of sponge/raspberry-like gold nanoclusters at the carbon rod. Russian J Electrochemistry. 2018;54:629-635. doi:10.1134/ S1023193518080037

111. He Q, Tian Y, Wu Y, et al. Electrochemical sensor for rapid and sensitive detection of tryptophan by a $\mathrm{Cu}_{2} \mathrm{O}$ nanoparticles-coated reduced graphene oxide nanocomposite. Biomolecules. 2019;9 (5):176. doi:10.3390/biom9050176

112. Gupta A, Jamatia R, Patil RA, Ma Y-R, Pal AK. Copper oxide/ reduced graphene oxide nanocomposite-catalyzed synthesis of flavanones and flavanones with triazole hybrid molecules in one pot: a green and sustainable approach. ACS Omega. 2018;3 (7):7288-7299. doi:10.1021/acsomega.8b00334

113. Zhang Q, Zhang $\mathrm{K}, \mathrm{Xu} \mathrm{D}$, et al. $\mathrm{CuO}$ nanostructures: synthesis, characterization, growth mechanisms, fundamental properties, and applications. Prog Mater Sci. 2014;60:208-337.

114. Devi AB, Moirangthem DS, Talukdar NC, Devi MD, Singh NR, Luwang MN. Novel synthesis and characterization of $\mathrm{CuO}$ nanomaterials: biological applications. Chine Chem Lett. 2014;25 (12):1615-1619. doi:10.1016/j.cclet.2014.07.014
115. Hou L, Zhang C, Li L, et al. CO gas sensors based on p-type $\mathrm{CuO}$ nanotubes and $\mathrm{CuO}$ nanocubes: morphology and surface structure effects on the sensing performance. Talanta. 2018;188:41-49. doi:10.1016/j.talanta.2018.05.059

116. Lauriente DH, DeBoo A, Sakota K. Sulfuric Acid. Fisherprint Ltd;1999;1.

117. Ashar NG, Golwalkar KR. A Practical Guide to the Manufacture of Sulfuric Acid, Oleums, and Sulfonating Agents. Springer; 2013.

118. Dheepthi GunaVathana S, Wilson J, Prashanthi R, Cyrac Peter A. $\mathrm{CuO}$ nanoflakes anchored polythiophene nanocomposite: voltammetric detection of L-Tryptophan. Inorg Chem Commun. 2021;124:108398. doi:10.1016/j.inoche.2020.108398

119. Molaakbari E, Mostafavi A, Beitollahi H. Simultaneous electrochemical determination of dopamine, melatonin, methionine and caffeine. Sens Actuators B Chem. 2015;208:195-203. doi:10.1016/j.snb.2014.10.130

120. Bagheri H, Afkhami A, Hashemi P, Ghanei M. Simultaneous and sensitive determination of melatonin and dopamine with $\mathrm{Fe}_{3} \mathrm{O}_{4}$ nanoparticle-decorated reduced graphene oxide modified electrode. RSC Adv. 2015;5(28):21659-21669. doi:10.1039/ C4RA16802J

121. Rao ML, Gross G, Strebel B, et al. Circadian rhythm of tryptophan, serotonin, melatonin, and pituitary hormones in schizophrenia. Biol Psychiatry. 1994;35(3):151-163. doi:10.1016/0006-3223 (94)91147-9

122. Comai S, Bertazzo A, Carretti N, Podfigurna-Stopa A, Luisi S, Costa CVL. Serum levels of tryptophan, 5-hydroxytryptophan and serotonin in patients affected with different forms of amenorrhea. Int $J$ Tryptophan Res. 2010;3:69-75. doi:10.4137/IJTR.S3804

123. Tan D-X, Manchester LC, Sanchez-Barcelo E, Mediavilla MD, Reiter RJ. Significance of high levels of endogenous melatonin in mammalian cerebrospinal fluid and in the central nervous system. Curr Neuropharmacol. 2010;8(3):162-167. doi:10.2174/ 157015910792246182

124. Thomas A, Kumar KG. Acetylene black-chitosan mediated electro-oxidation of serotonin and melatonin: an efficient platform for simultaneous voltammetric sensing. Ionics. 2019;25(5):23372349.

125. Anithaa AC, Asokan K, Lavanya N, Sekar C. Nicotinamide adenine dinucleotide immobilized tungsten trioxide nanoparticles for simultaneous sensing of norepinephrine, melatonin and nicotine. Biosens Bioelectron. 2019;143:111598. doi:10.1016/j. bios.2019.111598

126. Ali I, Soomro B, Omanovic S. Electrochemical regeneration of NADH on a glassy carbon electrode surface: the influence of electrolysis potential. Electrochem commun. 2011;13(6):562565. doi:10.1016/j.elecom.2011.03.010

127. Harrell CR, Djonov V, Fellabaum C, Volarevic V. Risks of using sterilization by gamma Radiation: the other side of the coin. Int J Med Sci. 2018;15(3):274-279. doi:10.7150/ijms.22644

128. Camargo JR, Andreotti IAA, Kalinke C, Henrique JM, Bonacin JA, Janegitz BC. Waterproof paper as a new substrate to construct a disposable sensor for the electrochemical determination of paracetamol and melatonin. Talanta. 2020;208:120458. doi:10.1016/j. talanta.2019.120458

129. Vasapollo G, Sole RD, Mergola L, et al. Molecularly imprinted polymers: present and future prospective. Int J Mol Sci. 2011;12 (9):5908-5945. doi:10.3390/ijms12095908

130. Gupta P, Goyal RN. Graphene and Co-polymer composite based molecularly imprinted sensor for ultratrace determination of melatonin in human biological fluids. RSC $A d v$. 2015;5(50):4044440454. doi:10.1039/C5RA04942C

131. Chen L, Wang X, Lu W, Wu X, Li J. Molecular imprinting: perspectives and applications. Chem Soc Rev. 2016;45(8):21372211. 
132. Verheyen E, Schillemans JP, van Wijk M, Demeniex M-A, Hennink WE, van Nostrum CF. Challenges for the effective molecular imprinting of proteins. Biomaterials. 2011;32 (11):3008-3020. doi:10.1016/j.biomaterials.2011.01.007

133. Han J, Zhao J, Li Z, et al. Nanoporous carbon derived from dandelion pappus as an enhanced electrode material with low cost for amperometric detection of tryptophan. $J$ Electroanal Chem. 2018;818:149-156. doi:10.1016/j.jelechem.2018.04.044

134. Deegan RD, Bakajin O, Dupont TF, Huber G, Nagel SR, Witten TA. Capillary flow as the cause of ring stains from dried liquid drops. Nature. 1997;389(6653):827-829. doi:10.1038/39827

135. Kaliyaraj Selva Kumar A, Zhang Y, Li D, Compton RG. A minireview: how reliable is the drop casting technique? Electrochem commun. 2020;121(106867). doi:10.1016/j.elecom.2020.106867

136. Shaidarova LG, Chelnokova IA, Makhmutova GF, Degteva MA, Gedmina AV, Budnikov HC. Flow-injection and sequential injection determination of hydroxypurines on an electrode modified with mixed-valence ruthenium and iridium oxides. $J$ Analytical Chem. 2014;69(9):864-874. doi:10.1134/S106193481409010X

137. Konz W. Integrated Total Analysis Systems. Comprehensive Sampling and Sample Preparation. Academic Press; 2012:99107.

138. Shaidarova LG, Chelnokova IA, Il'ina MA, Leksina YA, Budnikov HC. Amperometric detection of tryptophan and pyridoxine on a dual screen-printed electrode modified by gold nanoparticles in a flow-injection system. J Analytical Chem. 2019;74 (6):584-590. doi:10.1134/S106193481906011X

139. Nguyen N-T, Wu Z. Micromixers - a review. J Micromechanics Microengineering. 2005;15:1. doi:10.1088/0960-1317/15/2/R0

140. Jeon H, Khan A. Label-free electrochemical microfluidic chip for the antimicrobial susceptibility testing. Antibiotics. 2020;9 (6):348. doi:10.3390/antibiotics9060348

141. Gomez FJ, Martín A, Silva MF, Escarpa A. Microchip electrophoresis-single wall carbon nanotube press-transferred electrodes for fast and reliable electrochemical sensing of melatonin and its precursors. Electrophoresis. 2015;36(16):1880-1885. doi:10.1002/elps.201400580

142. Escarpa A. Lights and shadows on Food Microfluidics. Lab Chip. 2014;14(17):3213-3224. doi:10.1039/C4LC00172A

143. Escarpa A. Food electroanalysis: sense and simplicity. Chem Record. 2012;12(1):72-91. doi:10.1002/tcr.201100033

144. Ragab MAA, El-Kimary EI. Recent advances and applications of microfluidic capillary electrophoresis: a comprehensive review (2017-Mid 2019). Critical Rev Analytical Chem. 2020;1:33. doi:10.1080/10408347.2020.1765729

145. Stefan-van Staden R-I, Gugoasa LA, Socaci C, Biris AR. New nanocomposite-graphene pastes based stochastic microsensors. RSC Adv. 2015;5(81):66185-66191. doi:10.1039/C5RA13054A

146. Movileanu L. Interrogating single proteins through nanopores: challenges and opportunities. Trends Biotechnol. 2009;27 (6):333-341. doi:10.1016/j.tibtech.2009.02.008

147. Schmidt J. Stochastic sensors. J Mater Chem. 2005;15(8):831840. doi:10.1039/b414551h

148. Ilie-Mihai R-M, Stefan-van Staden R-I, Magerusan L, Coros M, Pruneanu S. Enantioanalysis of tryptophan in whole blood samples using stochastic sensors - a screening test for gastric cancer. Chirality. 2020;32(2):215-222. doi:10.1002/chir.23155

149. Bayley H, Cremer PS. Stochastic sensors inspired by biology. Nature. 2001;413(6852):226-230. doi:10.1038/35093038

150. Stefan-van Staden R-I, Lungu-Moscalu A, van Staden JF. Pattern recognition of melatonin using stochastic sensors. $N \mathrm{~J}$ Chem. 2019;43(13):5196-5201. doi:10.1039/C9NJ00588A

151. Wu Y, Wang T, Zhang C, Xing X-H. A rapid and specific colorimetric method for free tryptophan quantification. Talanta. 2018;176:604-609. doi:10.1016/j.talanta.2017.08.002
152. Xu L, Yan $\mathrm{W}, \mathrm{Ma} \mathrm{W}$, et al. SERS encoded silver pyramids for attomolar detection of multiplexed disease biomarkers. $A d v$ Mater. 2015;27(10):1706-1711. doi:10.1002/adma.201402244

153. Cortés E, Etchegoin PG, Le Ru EC, Fainstein A, Vela ME, Salvarezza RC. Strong correlation between molecular configurations and charge-transfer processes probed at the single-molecule level by surface-enhanced raman scattering. $J$ Am Chem Soc. 2013;135(7):2809-2815. doi:10.1021/ja312236y

154. Min F, Dechan L, Ruiyun Y, et al. Highly sensitive detection of tryptophan (Trp) in serum based on diazo-reaction coupling with Surface-Enhanced Raman Scattering and colorimetric assay. Anal Chim Acta. 2020;1119(1):52-59. doi:10.1016/j.aca.2020.04.039

155. Vorhees CV, Butcher RE, Brunner RL, Wootten V. Developmental toxicity and psychotoxicity of sodium nitrite in rats. Food Chem Toxicol. 1984;22(1):1-6. doi:10.1016/0278-6915(84)90044-9

156. Neth MR, Love JS, Horowitz BZ, Shertz MD, Sahni R, Daya MR. Fatal sodium nitrite poisoning: key considerations for prehospital providers. Prehospital Em Care. 2020;1-7. doi:10.1080/ 10903127.2020.1838009

157. Wu D, Yu Y, Zhang J, Guo L, Kong Y. Chiral poly(ionic liquid) with nonconjugated backbone as a fluorescent enantioselective sensor for phenylalaninol and tryptophan. ACS Appl Mater Interfaces. 2018;10 (27):23362-23368. doi:10.1021/acsami.8b04869

158. Pundi A, Chang C-J, Chen J, Hsieh S-R, Lee M-C. A chiral carbazole based sensor for sequential "on-off-on" fluorescence detection of $\mathrm{Fe}^{3+}$ and tryptophan/histidine. Sens Actuators B Chem. 2021;328:129084. doi:10.1016/j.snb.2020.129084

159. Jafari M, Tashkhourian J, Absalan G. Chiral recognition of tryptophan enantiomers using chitosan-capped silver nanoparticles: scanometry and spectrophotometry approaches. Talanta. 2018;178:870-878. doi:10.1016/j.talanta.2017.10.005

160. Peveler WJ, Roldan A, Hollingsworth N, Porter MJ, Parkin IP. Multichannel detection and differentiation of explosives with a quantum dot array. ACS Nano. 2016;10(1):1139-1146. doi:10.1021/acsnano.5b06433

161. He Y, He X, Liu X, Gao L, Cui H. Dynamically tunable chemiluminescence of luminol-functionalized silver nanoparticles and its application to protein sensing arrays. Anal Chem. 2014;86 (24):12166-12171. doi:10.1021/ac503123q

162. $\mathrm{Xu} \mathrm{S}$, Lu X, Yao $\mathrm{C}$, et al. A visual sensor array for pattern recognition analysis of proteins using novel blue-emitting fluorescent gold nanoclusters. Anal Chem. 2014;86(23):11634-11639. doi:10.1021/ac502643s

163. He Y, Xu B, Li W, Yu H. Silver nanoparticle-based chemiluminescent sensor array for pesticide discrimination. J Agric Food Chem. 2015;63(11):2930-2934. doi:10.1021/acs.jafc.5b00671

164. Zhang D, Liu J, Jiang C, Liu A, Xia B. Quantitative detection of formaldehyde and ammonia gas via metal oxide-modified graphenebased sensor array combining with neural network model. Sens Actuators B Chem. 2017;240:55-65. doi:10.1016/j.snb.2016.08.085

165. Huang W, Deng Y, He Y. Visual colorimetric sensor array for discrimination of antioxidants in serum using $\mathrm{MnO}_{2}$ nanosheets triggered multicolor chromogenic system. Biosens Bioelectron. 2017;91:89-94. doi:10.1016/j.bios.2016.12.028

166. Huang W, Xie Z, Deng Y, He Y. 3,3',5,5'-tetramethylbenzidinebased quadruple-channel visual colorimetric sensor array for highly sensitive discrimination of serum antioxidants. Sens Actuators B Chem. 2018;254:1057-1060. doi:10.1016/j. snb.2017.08.005

167. Liu B, You Y, Lin D, Chen Z, Qiu P. Simple colorimetric and fluorometric assay based on 2,3-naphthalenedialdehyde for melatonin in human saliva. Chem Africa. 2020;3(1):181-188. doi: $10.1007 / \mathrm{s} 42250-019-00105-7$

168. Guo B, Liu X, Tan X. Acidizing. In: Guo B, Liu X, Tan X. Petroleum Production Engineering (Second Edition). Gulf Professional Publishing; 2017:367-387. 
169. Matsufuji H, Matsui T, Oki T, Osajima Y, Kawasaki T. Fluorometric determination of angiotensins in human plasma derivatized with 2, 3-naphthalenedialdehyde. Bunseki Kagaku. 1995;44(10):783-788. doi:10.2116/bunsekikagaku.44.783

170. LoPachin RM, Gavin T. Reactions of electrophiles with nucleophilic thiolate sites: relevance to pathophysiological mechanisms and remediation. Free Radic Res. 2016;50(2):195-205. doi:10.3109/10715762.2015.1094184

171. Chen Z, Zhou K, Ha W, et al. Development of a low-cost, simple, fast and quantitative lateral-flow immunochromatographic assay (ICA) strip for melatonin in health foods. Food Agric Immunol. 2019;30(1):497-509. doi:10.1080/09540105.2019.1602112

172. Liu N, Xu Z, Morrin A, Luo X. Low fouling strategies for electrochemical biosensors targeting disease biomarkers. Analytical Methods. 2019;11(6):702-711. doi:10.1039/ C8AY02674B

173. Song Z, Sheng G, Cui Y, et al. Low fouling electrochemical sensing in complex biological media by using the ionic liquiddoped conducting polymer PEDOT: application to voltammetric determination of dopamine. Microchimica Acta. 2019;186(4):220. doi:10.1007/s00604-019-3340-x

174. Kim S, Lee S, Park J, Lee JY. Electrochemical co-deposition of polydopamine/hyaluronic acid for anti-biofouling bioelectrodes. Front Chem. 2019;7:262. doi:10.3389/fchem.2019.00262

175. Cao B, Lee C-J, Zeng Z, et al. Electroactive poly(sulfobetaine3,4-ethylenedioxythiophene) (PSBEDOT) with controllable antifouling and antimicrobial properties. Chem Sci. 2016;7(3):19761981. doi:10.1039/C5SC03887A

176. Walker WH, Walton JC, DeVries AC, Nelson RJ. Circadian rhythm disruption and mental health. Transl Psychiatry. 2020;10 (1):28. doi:10.1038/s41398-020-0694-0

177. Lee E, Kim M. Light and life at night as circadian rhythm disruptors. Chronobiol Med. 2019;1(3):95-102. doi:10.33069/ cim.2019.0016
178. Ahmed KZ. Photo period and ovarian physiology a chronobiological approach in zebra fish Danio rerio. Shodhganga. 2018.

179. Khan ZA, Labala RK, Yumnamcha T, et al. Artificial light at night (ALAN), an alarm to ovarian physiology: a study of possible chronodisruption on zebrafish (Danio rerio). Sci Total Environ. 2018;628:1407-1421. doi:10.1016/j.scitotenv.2018.02.101

180. Shneider A, Kudriavtsev A, Vakhrusheva A. Can melatonin reduce the severity of COVID-19 pandemic? Int Rev Immunol. 2020;1-10.

181. Kleszczyński K, Slominski AT, Steinbrink K, Reiter RJ. Clinical trials for use of melatonin to fight against COVID-19 are urgently needed. Nutrients. 2020;12(9):2561. doi:10.3390/nu12092561

182. Belladonna ML, Orabona C. Potential benefits of tryptophan metabolism to the efficacy of tocilizumab in COVID-19. Front Pharmacol. 2020;11:959. doi:10.3389/fphar.2020.00959

183. Essa MM, Hamdan H, Chidambaram SB, et al. Possible Role of Tryptophan and Melatonin in COVID-19. London, England: SAGE Publications Sage UK; 2020.

184. Jo Y, Oh C, Lee HJ. Microelectromechanical systems-based neurotools for non-invasive ultrasound brain stimulation. Chronobiol Med. 2019;1(2):55-59. doi:10.33069/cim.2019.0009

185. Lee H-J. Chronobiology, the future of medicine. Chronobiol Med. 2019;1(1):1-2. doi:10.33069/cim.2019.0006

186. Heikenfeld J, Jajack A, Rogers J, et al. Wearable sensors: modalities, challenges, and prospects. Lab Chip. 2018;18(2):217-248. doi:10.1039/C7LC00914C

187. Nguyen V-T, Song S, Park S, Joo C. Recent advances in highsensitivity detection methods for paper-based lateral-flow assay. Biosens Bioelectron. 2020;152:112015. doi:10.1016/j. bios.2020.112015
International Journal of Nanomedicine

\section{Publish your work in this journal}

The International Journal of Nanomedicine is an international, peerreviewed journal focusing on the application of nanotechnology in diagnostics, therapeutics, and drug delivery systems throughout the biomedical field. This journal is indexed on PubMed Central, MedLine, CAS, SciSearch ${ }^{\mathbb{}}$, Current Contents ${ }^{\mathbb{R}} /$ Clinical Medicine, $^{-}$

\section{Dovepress}

Journal Citation Reports/Science Edition, EMBase, Scopus and the Elsevier Bibliographic databases. The manuscript management system is completely online and includes a very quick and fair peer-review system, which is all easy to use. Visit http://www.dovepress.com/ testimonials.php to read real quotes from published authors. 